\title{
Stacked OSTBC: Error Performance and Rate Analysis
}

\author{
Aydin Sezgin, Member, IEEE and Oliver Henkel, Member, IEEE
}

\begin{abstract}
It is well known, that the Alamouti scheme is the only space-time code from orthogonal design achieving the capacity of a multiple-input multiple-output (MIMO) wireless communication system with $n_{T}=2$ transmit antennas and $n_{R}=1$ receive antenna. In this work, we propose the $n$-times stacked Alamouti scheme for $n_{T}=2 n$ transmit antennas and show that this scheme achieves the capacity in the case of $n_{R}=1$ receive antenna. This result may regarded as an extension of the Alamouti case. For the more general case of more than one receive antenna, we show that if the number of transmit antennas is higher than the number of receive antennas we achieve a high portion of the capacity with this scheme. Further, we show that the MIMO capacity is at most twice the rate achieved with the proposed scheme for all SNR. We derive lower and upper bounds for the rate achieved with this scheme and compare it with upper and lower bounds for the capacity. In addition to the capacity analysis based on the assumption of a coherent channel, we analyze the error rate performance of the stacked OSTBC with the optimal ML detector and with the suboptimal latticereduction (LR) aided zero-forcing detector. We compare the error rate performance of the stacked OSTBC with spatial multiplexing (SM) and full-diversity achieving schemes. Finally, we illustrate the theoretical results by numerical simulations.
\end{abstract}

\section{INTRODUCTION}

Recent information theoretic results have demonstrated that the ability of a system to support a high link quality and higher data rates in the presence of Rayleigh fading improves significantly with the use of multiple transmit and receive antennas [1], [2]. Since then there has been considerable work on a variety of schemes [3] which exploit multiple antennas at both the transmitter and receiver in order to either obtain transmit and receive diversity and therefore increase the reliability of the system, e.g., orthogonal space-time block codes (OSTBC) and space-time trellis codes [4]-[6] or achieve the theoretical bounds [7] derived in [1], [2]. Interested readers are referred to [3], where a detailed analysis of different schemes is given.

The performance of OSTBC with respect to mutual information has been analyzed (among others) in [8]-[11] and it was shown that the capacity is achieved only in the case of $n_{T}=2$ transmit, the well known Alamouti scheme [5], and

Manuscript received March 29, 2006; revised July 10, 2006. This paper was presented in part at the VTC 2004-Fall, Los Angeles, September 2004. The associate editor coordinating the review of this manuscript and approving it for publication was Dr. R. Michael Buehrer.

A.Sezgin has been with the Fraunhofer-Institute for Telecommunications, Heinrich-Hertz-Institut, Einsteinufer 37, D-10587 Berlin, Germany. He is now with the Information Systems Laboratory, Stanford University, CA 943059510, USA (e-mail:sezgin@stanford.edu).

O.Henkel is with the Fraunhofer-Institute for Telecomm., HHI, Einsteinufer 37, 10587 Berlin, Germany, (e-mail:henkel@hhi.de).
$n_{R}=1$ receive antennas due to the rate loss inherent in OSTBC with higher number of transmit antennas. Recently, it was shown in [12] that due to this rate loss, OSTBC with odd number of antennas are always outperformed by OSTBC with even number of antennas, restricting even more the deployment of OSTBC. On the one hand, we have the OSTBC with low complexity and low rates. On the other hand, we have the space-time trellis codes, which achieve higher spectral efficiency in addition to high performance with respect to frame error rates. However, the decoding complexity of space-time trellis codes is increasing exponentially with the number of transmit antennas and the transmission rate. In order to achieve higher spectral efficiency combined with low complexity maximum likelihood detectors, [13]-[17] designed quasi-orthogonal space-time block codes (QSTBC) with transmission rate one for more than two transmit antennas.

Other approaches aimed at reducing the decoding complexity of space-time trellis codes. For instance, a layered spacetime architecture was proposed in [18], where the transmit antennas were partitioned into two-antenna groups and on each group space-time trellis codes were used as component codes. In order to further decrease the complexity of this layered space-time architecture, [19]-[21] used the Alamouti scheme as component code for each group in combination with a suboptimal successive group interference suppression detection strategy. The outage probability of this scheme was analyzed in [22] for $n_{T}>n_{R}$ and an upper bound was derived. An asymptotic analysis of the rate achievable with this scheme is performed in [23]. For $n=2$, this transmission scheme is also referred to as double-space-time transmit diversity (DSTTD) and was proposed as one possible candidate for high speed downlink packet access (HSDPA) in 3GPP and beyond [24].

It is obvious that reducing the computational complexity of the detector without sacrificing much performance is an important issue. There is a huge amount of suboptimal detectors with low complexity in the literature, linear detectors like zero-forcing $(\mathrm{ZF})$ or minimum mean square error (MMSE) and nonlinear detectors like e.g. VBLAST [25]. Unfortunately, these detectors significantly sacrifice performance in terms of bit-error-rate (BER). Recently, lattice reduction (LR) aided detection in combination with suboptimal detectors was proposed by Yao and Wornell in order to improve the performance of multi antenna systems [26]. The lattice reduction algorithm proposed in [26] is optimal, but works only for MIMO systems with two transmit and two receive antennas. The impact of receive antenna correlation on the performance of LR-aided detection was analyzed in [27]. In [28], the work of [26] was extended to systems with more transmit and receive antennas, 
using the sub-optimal LLL [29] lattice reduction algorithm. In [30], the LR-aided schemes in [28] were adopted to the MMSE criterion. Note that the error rate curves of all these LR detectors are parallel to those for maximum likelihood (ML) detection with only some penalty in power efficiency.

In this work, we show that the stacked Alamouti scheme is capable to achieve the capacity in combination with the optimal maximum likelihood detector for the case of $n_{T}=2 n$ transmit antennas and $n_{R}=1$ receive antennas. This was also shown for the basic Alamouti scheme with $n_{T}=2$ and $n_{R}=$ 1 [8]. Our result may therefore be regarded as an extension of the Alamouti scheme to $n_{T}>2$. Furthermore, we show that in the case of more than one receive antenna and if $n_{T}>n_{R}$ the stacked Alamouti scheme is capable to achieve a significant portion of the capacity and approaches the capacity if $n_{T} \gg$ $n_{R}$. For any $n_{T}, n_{R}$, we show that the MIMO capacity is at most twice the rate achieved with the proposed scheme for all SNR. However, achieving high portions of the capacity does not guarantee good performance in terms of error probability. Thus, we compare the error-rate performance of the proposed scheme with spatial multiplexing (SM), a rate oriented spacetime transmission schemes which achieve a high portion of the capacity of MIMO systems, and with the aforementioned diversity-oriented QSTBC by deploying LR-aided linear ZF and ML detectors at the receiver, respectively.

The remainder of this paper is organized as follows. In Section [I] we introduce the system model and establish the notation. The structure of the stacked Alamouti scheme and the equivalent channel model are shown in section [III The analysis of the mutual information is presented in section IV LR-aided linear ZF detection is shortly described in section $\nabla$ including the analysis of the probability density function of the condition number of the equivalent channel generated by the different transmission schemes (SM,QSTBC, and stacked OSTBC). Section VI provides simulation results, followed by some concluding remarks in Section VII

\section{SySTEM MODEL}

We consider a system with $n_{T}$ transmit and $n_{R}$ receive antennas. Our system model is defined by

$$
\mathbf{Y}=\mathbf{G}_{n_{T}} \mathbf{H}^{T}+\mathbf{N}
$$

where $\mathbf{G}_{n_{T}}$ is the $\left(T \times n_{T}\right)$ transmit matrix, $\mathbf{Y}=$ $\left[\mathbf{y}_{1}, \ldots, \mathbf{y}_{n_{R}}\right]$ is the $\left(T \times n_{R}\right)$ receive matrix, $\mathbf{H}=$ $\left[\mathbf{h}_{1}, \ldots, \mathbf{h}_{n_{T}}\right]$ is a $\left(n_{R} \times n_{T}\right)$ matrix characterizing the coherent channel, and $\mathbf{N}=\left[\mathbf{n}_{1}, \ldots, \mathbf{n}_{n_{R}}\right]$ is the complex $\left(T \times n_{R}\right)$ white Gaussian noise (AWGN) matrix, where an entry $\left\{n_{t i}\right\}$ of $\mathbf{N}\left(1 \leq i \leq n_{R}\right)$ denotes the complex noise at the $i$ th receiver for a given time $t(1 \leq t \leq T)$. The real and imaginary parts of $n_{t i}$ are independent and $\mathcal{N}\left(0, n_{T} /(2 \mathrm{SNR})\right)$ distributed. An entry of the channel matrix is denoted by $\left\{h_{i j}\right\}$. This represents the complex gain of the channel between the $j$ th transmit $\left(1 \leq j \leq n_{T}\right)$ and the $i$ th receive $\left(1 \leq i \leq n_{R}\right)$ antenna, where the real and imaginary parts of the channel gains are independent and normal distributed random variables with $\mathcal{N}(0,1 / 2)$ per dimension. The channel matrix is assumed to be constant for a block of $T$ symbols and changes independently from block to block. The average power of the symbols transmitted from each antenna is normalized to be $1 / n_{T}$, so that the average power of the received signal at each receive antenna is one and the signal-to-noise ratio (SNR) is $\rho$. It is further assumed that the transmitter has no channel state information (CSI) and the receiver has perfect CSI.

\section{Code construction}

A space time block code is defined by its transmit matrix $\mathbf{G}_{n_{T}}$ with entries $\left\{x_{j}\right\}_{j=1}^{p}$, which are elements of the vector $\mathbf{x}=\left[x_{1}, \ldots, x_{p}\right]^{T}$ with $x_{1}, \ldots, x_{p} \in \mathcal{C}$, where $\mathcal{C} \subseteq \mathbb{C}$ denotes a complex modulation signal set with unit average power, e.g. $M$-PSK.. The rate $R$ of a space-time code is defined as $R=p / T$. In this paper, we focus on the rate $n_{T} / 2$ stacked Alamouti scheme. Starting with the well known (basic) Alamouti scheme [5] for $n_{T}=2$ transmit antennas

$$
\mathbf{G}_{2}\left(x_{1}, x_{2}\right)=\left[\begin{array}{rr}
x_{1} & x_{2} \\
x_{2}^{*} & -x_{1}^{*}
\end{array}\right] \text {, }
$$

the transmit matrix of the stacked Alamouti scheme with $n_{T}=$ $2 n$ is constructed in the following way

$$
\begin{aligned}
& \mathbf{G}_{n_{T}}\left(\left\{x_{j}\right\}_{j=1}^{n_{T}}\right) \\
& =\left[\mathbf{G}_{2}\left(x_{1}, x_{2}\right), \mathbf{G}_{2}\left(x_{3}, x_{4}\right), \ldots, \mathbf{G}_{2}\left(x_{n_{T}-1}, x_{n_{T}}\right)\right] .
\end{aligned}
$$

Example 3.1: For the case of $n=2$, i.e. $n_{T}=4$ transmit antennas we have

$$
\mathbf{G}_{4}\left(\left\{x_{j}\right\}_{j=1}^{4}\right)=\left[\begin{array}{rrrr}
x_{1} & x_{2} & x_{3} & x_{4} \\
x_{2}^{*} & -x_{1}^{*} & x_{4}^{*} & -x_{3}^{*}
\end{array}\right],
$$

which is also referred to as DSTTD [24].

After some manipulations (particularly complexconjugating) the system model in (1) can be rewritten as

$$
\mathbf{y}^{\prime}=\mathbf{H}^{\prime} \mathbf{x}+\mathbf{n}^{\prime}
$$

where $\mathbf{y}^{\prime}, \mathbf{n}^{\prime} \in \mathbb{C}^{2 n_{R}}$ and $\mathbf{H}^{\prime} \in \mathbb{C}^{2 n_{R} \times n_{T}}$. The equivalent channel equals

$$
\mathbf{H}^{\prime}=\left[\left(\mathbf{H}_{1}^{\prime}\right)^{T}, \ldots,\left(\mathbf{H}_{i}^{\prime}\right)^{T}, \ldots,\left(\mathbf{H}_{n_{R}}^{\prime}\right)^{T}\right]^{T},
$$

where $\mathbf{H}_{i}^{\prime}$ is given as

$$
\mathbf{H}_{i}^{\prime}=\left[\mathbf{H}_{i, 1}^{\prime}, \mathbf{H}_{i, 3}^{\prime}, \ldots, \mathbf{H}_{i, n_{T}-1}^{\prime}\right],
$$

where

$$
\mathbf{H}_{i, j}^{\prime}=\left[\begin{array}{cc}
h_{i j} & h_{i(j+1)} \\
-h_{i(j+1)}^{*} & h_{i j}^{*}
\end{array}\right] .
$$

\section{Mutual Information}

The instantaneous capacity $I$ of a MIMO system with $n_{T}$ transmit and $n_{R}$ receive antennas is given as [1], [2]

$$
I=\log _{2} \operatorname{det}\left(\mathbf{I}_{n_{T}}+\frac{\rho}{n_{T}} \mathbf{H}^{H} \mathbf{H}\right) .
$$

In the following two subsections, we derive lower and upper bounds for both the ergodic capacity and the average rate achievable with the proposed stacked scheme in order to yield lower and upper bounds on the ratio of the ergodic capacity to the average rate of the stacked OSTBC. In the third subsection, we characterize the absolute loss of the average rate of the stacked OSTBC to the ergodic capacity. 
A. Upper bounds on the ergodic capacity and the average rate of stacked OSTBC

By applying the trace-determinant inequality $\operatorname{det}(\mathbf{A})^{1 / n} \leq$ $\frac{1}{n} \operatorname{tr}(\mathbf{A})$, we arrive at a simple upper bound on the instantaneous capacity given as

$$
I \leq I_{u b}=L \log _{2}(1+\frac{\rho}{n_{T} L} \underbrace{\sum_{j=1}^{n_{T}} \sum_{i=1}^{n_{R}}\left|h_{j i}\right|^{2}}_{\lambda}),
$$

where $L$ is equal to $L=\min \left(n_{T}, n_{R}\right)$. Averaging the upper bound in (5) over all channel realizations results in [31] $(C=$ $\mathbb{E}[I]$ denotes ergodic capacity)

$$
\begin{aligned}
C \leq C_{u b}=\mathbb{E}\left[I_{u b}\right]= & \frac{L}{\ln (2)} \sum_{k=0}^{n_{T} n_{R}-1}\left(\frac{n_{T} L}{\rho}\right)^{n_{T} n_{R}-k-1} \\
& e^{\frac{n_{T} L}{\rho}} \Gamma\left(1-\left(n_{T} n_{R}-k\right), \frac{n_{T} L}{\rho}\right) .
\end{aligned}
$$

Note that for high SNR, the slope of the upper bound is equal to $L$. In addition to this upper bound, we compare the rate achieved with the stacked scheme with the following upper bound

$$
C \leq C_{\text {Jen }}=\log _{2}\left(\sum_{i=0}^{L}\left(\begin{array}{c}
L \\
i
\end{array}\right) \frac{K !}{(K-i) !}\left(\frac{\rho}{n_{T}}\right)^{i}\right),
$$

derived in [32] by using Jensen's inequality, where $K=$ $\max \left(n_{T}, n_{R}\right)$.

In the following, we analyze the performance of the stacked scheme with respect to mutual information and derive upper bounds for the average rate of the stacked scheme. We first analyze the case of $n_{R}=1$ receive antennas and then generalize the analysis to the case of arbitrary number of receive antennas.

1) Case $n_{R}=1$ : In case of $n_{R}=1$, the achievable rate of the stacked Alamouti scheme is

$$
I_{s A}=\frac{1}{2} \log _{2} \operatorname{det}\left(\mathbf{I}_{n_{T}}+\frac{\rho}{n_{T}}\left(\mathbf{H}_{1}^{\prime}\right)^{H} \mathbf{H}_{1}^{\prime}\right) .
$$

Using the determinant equality $\operatorname{det}(\mathbf{I}+\mathbf{A B})=\operatorname{det}(\mathbf{I}+\mathbf{B A})$, after some manipulations we arrive at

$$
I_{s A}=\log _{2}\left(1+\frac{\rho}{n_{T}} \sum_{j=1}^{n_{T}}\left|h_{j 1}\right|^{2}\right)
$$

which equals the capacity of a MIMO system with $n_{T}$ transmit and $n_{R}=1$ receive antennas [1], i.e. as long as $n_{R}=$ 1 , the capacity is achieved for arbitrary $n=n_{T} / 2$. Note that in [3, p.199] a Taylor series expansion is performed for the capacity and the mutual information achievable with certain schemes such as the stacked OSTBC. After comparing the first two expansion coefficients (the linear term and the second order coefficients) it is shown that the stacked OSTBC reaches second-order capacity for $n_{R}=1$, i.e. the secondorder coefficient of the mutual information of the stacked OSTBC is equal to the second-order coefficient of the capacity. Although essential features of the mutual information can be already seen from the first and second-order coefficients (especially at low SNR), our result above may regarded as more general, since the exact capacity and mutual information expressions are analyzed. Further note that the result above may be regarded as an extension of the results in [8]. There it was shown, that the basic Alamouti scheme with $n_{T}=2$ and $n_{R}=1$ achieves the capacity.

2) Case of $n_{T}=4$ and $n_{R}=2$ (DSTTD): In the case of $n_{T}=4$ transmit and $n_{R}=2$ receive antennas, the equivalent channel is given by

$$
\mathbf{H}^{\prime}=\left[\begin{array}{cccc}
h_{11} & h_{12} & h_{13} & h_{14} \\
-h_{12}^{*} & h_{11}^{*} & -h_{14}^{*} & h_{13}^{*} \\
h_{21} & h_{22} & h_{23} & h_{24} \\
-h_{22}^{*} & h_{21}^{*} & -h_{24}^{*} & h_{23}^{*}
\end{array}\right] .
$$

The achievable rate in this case is given as

$I_{s A}=\frac{1}{2} \log _{2} \operatorname{det}\left(\mathbf{I}_{n_{T}}+\frac{\rho}{n_{T}}\left[\begin{array}{cccc}\lambda_{1} & 0 & \alpha_{1} & \alpha_{2} \\ 0 & \lambda_{1} & -\alpha_{2}^{*} & \alpha_{1}^{*} \\ \alpha_{1}^{*} & -\alpha_{2} & \lambda_{2} & 0 \\ \alpha_{2}^{*} & \alpha_{1} & 0 & \lambda_{2}\end{array}\right]\right)$,

where $\lambda_{i}=\sum_{j=1}^{n_{T}}\left|h_{i j}\right|^{2}, \alpha_{1}=h_{11} h_{21}^{*}+h_{12} h_{22}^{*}+h_{13} h_{23}^{*}+$ $h_{14} h_{24}^{*}$, and $\alpha_{2}=-h_{11} h_{22}+h_{12} h_{21}-h_{13} h_{24}^{*}+h_{14} h_{23}$. Using Fischer's inequality

$$
\operatorname{det}\left(\left[\begin{array}{cc}
\mathbf{A} & \mathbf{B}^{\mathbf{H}} \\
\mathbf{B} & \mathbf{D}
\end{array}\right]\right) \leq \operatorname{det}(\mathbf{A}) \operatorname{det}(\mathbf{D})
$$

yields

$$
I_{s A} \leq \log _{2}\left(\left(1+\frac{\rho}{n_{T}} \lambda_{1}\right)\left(1+\frac{\rho}{n_{T}} \lambda_{2}\right)\right) .
$$

By using the arithmetic-geometric inequality, we arrive at

$$
I_{s A} \leq 2 \log _{2}\left(1+\frac{\rho}{2 n_{T}}\|\mathbf{H}\|^{2}\right) .
$$

This upper bound equals to twice the rate of a full code rate OSTBC for $n_{T}=4$ transmit and $n_{R}=2$ receive antennas with a power penalty of $3 \mathrm{~dB}$. In this particular case a more precise statement can be made due to the following strict form of Fischer's inequality [33]

Lemma 4.1: Let $\mathbf{P}=\left[\begin{array}{cc}\mathbf{A} & \mathbf{B}^{\mathbf{H}} \\ \mathbf{B} & \mathbf{D}\end{array}\right](\mathbf{A}, \mathbf{D}$ square, nonempty) be positive definite. Then

$$
\mathbf{B} \text { has full rank } \Rightarrow \operatorname{det} \mathbf{P}<(\operatorname{det} \mathbf{A})(\operatorname{det} \mathbf{D})
$$

Proof: Let $\mathbf{R} \succ 0$ denote positive definiteness, and $\mathbf{R} \succ$ $\mathbf{S}$ defined by $(\mathbf{R}-\mathbf{S}) \succ 0$. Then [34, 7.7.6] $\mathbf{P} \succ 0 \Leftrightarrow(\mathbf{A} \succ$ $\left.0, \mathbf{D} \succ \mathbf{B A}^{-1} \mathbf{B}^{H}\right)$. Thus for arbitrary $\mathbf{B}$ holds $\mathbf{D}-(\mathbf{D}-$ $\left.\mathbf{B A}^{-1} \mathbf{B}^{H}\right)=\mathbf{B} \mathbf{A}^{-1} \mathbf{B}^{H} \succeq 0$ and becomes strict if $\mathbf{B}$ has full rank. Since $(0 \prec \mathbf{S} \prec \mathbf{R} \Rightarrow \operatorname{det} \mathbf{S}<\operatorname{det} \mathbf{R})$ we obtain $\operatorname{det} \mathbf{P}=(\operatorname{det} \mathbf{A})\left(\operatorname{det}\left[\mathbf{D}-\mathbf{B} \mathbf{A}^{-1} \mathbf{B}^{H}\right]\right)<(\operatorname{det} \mathbf{A})(\operatorname{det} \mathbf{D})$, if $\mathbf{B}$ has full rank.

From det $\mathbf{B}=\left|\alpha_{1}\right|^{2}+\left|\alpha_{2}\right|^{2}$ it follows, that apart from the set of events $\left\{\alpha_{1}=\alpha_{2}=0\right\}$ of measure zero, $\mathbf{B}$ has full rank, thus the upper bound for $I_{s A}$ is strict with probability one. 
3) Case of arbitrary $n_{R}$ : The available portion of the mutual information achievable with $n_{R} \geq 1$ for the stacked Alamouti scheme is

$$
I_{s A}=\frac{1}{2} \log _{2} \operatorname{det}\left(\mathbf{I}_{n_{T}}+\frac{\rho}{n_{T}}\left(\mathbf{H}^{\prime}\right)^{H} \mathbf{H}^{\prime}\right) .
$$

Following the derivation above for arbitrary $n_{R}$ results in

$$
I_{s A} \leq I_{s A}^{u b}=\frac{L_{1}}{2} \log _{2}\left(1+\frac{2 \rho}{n_{T} L_{1}}\|\mathbf{H}\|^{2}\right),
$$

where $L_{1}=\min \left(n_{T}, 2 n_{R}\right)$. By averaging (10) over all channel realizations, an upper bound on the average rate $R_{s A}^{u b} \geq \mathbb{E}\left[I_{s A}\right]$ of the stacked Alamouti scheme similar to 6 may be obtained

$$
\begin{aligned}
R_{s A} \leq R_{s A}^{u b}=\mathbb{E}\left[I_{u b}\right]= & \frac{L_{1}}{2 \ln (2)} \sum_{k=0}^{n_{T} n_{R}-1}\left(\frac{n_{T} L_{1}}{2 \rho}\right)^{n_{T} n_{R}-k-1} \\
& e^{\frac{n_{T} L_{1}}{2 \rho}} \Gamma\left(1-\left(n_{T} n_{R}-k\right), \frac{n_{T} L_{1}}{2 \rho}\right),
\end{aligned}
$$

which can be approximated using $\log _{2}(1+x) \approx \log _{2}(x)$ for $x \gg 1$ by

$$
R_{s A}^{u b} \approx \frac{L_{1}}{2} \log _{2}\left(\frac{2 \rho}{n_{T} L_{1}}\right)+\frac{L_{1}}{2 \ln (2)}\left(\sum_{p=1}^{n_{T} n_{R}-1} \frac{1}{p}-\gamma\right) .
$$

Note that the approximation gets better for higher SNR and may be inaccurate for low SNR. Further note that, for high SNR, the slope of the upper bound (11) and its approximation is equal to $L_{1} / 2$.

\section{B. Lower bounds on the ergodic capacity and the average rate of stacked OSTBC}

Similarly to the last subsection, here we derive lower bounds for the ergodic capacity and the average rate of the stacked OSTBC. Due to the peculiar property of stacked OSTBC, lower bounds are obtained in the procedure for the following cases: (i) $n_{T} \leq n_{R}$, (ii) $n_{R}<n_{T}<2 n_{R}$, (iii) $2 n_{R} \leq n_{T} \leq$ $4 n_{R}$, and (iv) $4 n_{R}<n_{T}$.

First of all, from [35] we obtain the following lower bound on the ergodic capacity

$$
C \geq C_{l b}=\sum_{j=1}^{L} \log _{2}\left(1+\frac{\rho}{n_{T}} \exp \left(\sum_{p=1}^{K-j} \frac{1}{p}-\gamma\right)\right),
$$

where $\gamma \approx 0.57721566$ is Euler's constant.

In order to derive an upper bound on the ratio of the ergodic capacity to the average rate achieved with the stacked scheme, we need a lower bound for the average rate of the stacked scheme. To this end, we rewrite (9) as follows

$$
I_{s A}=\frac{1}{2} \log _{2} \operatorname{det}\left(\mathbf{I}_{n_{T}}+\frac{\rho}{n_{T}}(\mathbf{H})^{H} \mathbf{H}+\frac{\rho}{n_{T}}\left(\mathbf{H}_{e}^{\prime}\right)^{H} \mathbf{H}_{e}^{\prime}\right),
$$

where $\mathbf{H}$ is the actual MIMO channel, which is obtained by taking the odd rows of the equivalent channel $\mathbf{H}^{\prime}$ and $\mathbf{H}_{e}$ is obtained by taking the even rows of $\mathbf{H}^{\prime}$. The relation between the actual channel $\mathbf{H}$ and $\mathbf{H}_{e}$ is described in the following proposition.

Proposition 4.1: Let $\mathbf{H}_{e}$ be the even and $\mathbf{H}$ the odd rows of $\mathbf{H}^{\prime}$ given in (2), respectively. Then the following holds

1) $\mathbf{H}_{e}=\mathbf{H}^{*} \mathbf{J}$, where $\varepsilon^{1}$

$$
\mathbf{J}=\mathbf{I}_{\frac{n_{T}}{2}} \otimes\left[\begin{array}{cc}
0 & 1 \\
-1 & 0
\end{array}\right] .
$$

2) $\mathbb{E}\left[\mathbf{H H}_{e}^{H}\right]=\mathbb{E}\left[\mathbf{H J}^{T} \mathbf{H}^{T}\right]=\mathbf{0}$.

Proof: The proof is straightforward and uninformative and thus it is omitted.

Eq. (13) can be rewritten as

$$
\begin{aligned}
I_{s A} & =\frac{1}{2} \log _{2}\left(\operatorname{det}\left(\mathbf{I}_{n_{T}}+\frac{\rho}{n_{T}}(\mathbf{H})^{H} \mathbf{H}\right) \times\right. \\
& \left.\operatorname{det}\left(\mathbf{I}_{n_{T}}+\frac{\rho}{n_{T}} \mathbf{H}_{e}^{\prime}\left(\mathbf{I}_{n_{T}}+\frac{\rho}{n_{T}}(\mathbf{H})^{H} \mathbf{H}\right)^{-1}\left(\mathbf{H}_{e}^{\prime}\right)^{H}\right)\right) \\
& =\frac{1}{2} \log _{2} \operatorname{det}\left(\mathbf{I}_{n_{T}}+\frac{\rho}{n_{T}}(\mathbf{H})^{H} \mathbf{H}\right)+\frac{1}{2} \times \\
& \log _{2} \operatorname{det}\left(\mathbf{I}_{n_{T}}+\frac{\rho}{n_{T}} \mathbf{H}_{e}^{\prime}\left(\mathbf{I}_{n_{T}}+\frac{\rho}{n_{T}}(\mathbf{H})^{H} \mathbf{H}\right)^{-1}\left(\mathbf{H}_{e}^{\prime}\right)^{H}\right) .
\end{aligned}
$$

Since $\quad \mathbf{H}_{e}^{\prime}\left(\mathbf{I}_{n_{T}}+\frac{\rho}{n_{T}}(\mathbf{H})^{H} \mathbf{H}\right)^{-1}\left(\mathbf{H}_{e}^{\prime}\right)^{H} \quad$ is a positive semidefinite matrix, it follows immediately that the rate achieved with the stacked Alamouti is lower bounded by

$$
I_{s A} \geq \frac{1}{2} \log _{2} \operatorname{det}\left(\mathbf{I}_{n_{T}}+\frac{\rho}{n_{T}}(\mathbf{H})^{H} \mathbf{H}\right),
$$

which is half the capacity of a MIMO system with $n_{T}$ transmit and $n_{R}$ receive antennas.

Another lower bound is obtained for the case $n_{T} \leq n_{R}$ by applying Minkowski's determinant inequality [34, p.482] $\left(\operatorname{det}(\mathbf{A}+\mathbf{B}) \geq\left(\operatorname{det}(\mathbf{A})^{\frac{1}{n}}+\operatorname{det}(\mathbf{B})^{\frac{1}{n}}\right)^{n}, \mathbf{A} \succ 0, \mathbf{B} \succeq 0\right)$ to (9)

$$
\begin{aligned}
R_{s A} & =\mathbb{E}\left[\frac{1}{2} \log _{2} \operatorname{det}\left(\mathbf{I}+\frac{\rho}{n_{T}}\left(\mathbf{H}^{\prime}\right)^{H} \mathbf{H}^{\prime}\right)\right] \\
& \geq \frac{n_{T}}{2} \mathbb{E}\left[\log _{2}\left(1+\rho \operatorname{det}\left(\frac{1}{n_{T}}\left(\mathbf{H}^{\prime}\right)^{H} \mathbf{H}^{\prime}\right)^{\frac{1}{n_{T}}}\right)\right] \\
& =\frac{n_{T}}{2} \mathbb{E}\left[\log _{2}\left(1+\rho \operatorname{det}\left(\frac{1}{n_{T}}\left(\mathbf{H}^{H} \mathbf{H}+\mathbf{H}_{e}^{H} \mathbf{H}_{e}\right)\right)^{\frac{1}{n_{T}}}\right)\right] .
\end{aligned}
$$

Applying again Minkowski’s determinant inequality results in

$$
\begin{array}{r}
R_{s A} \geq \frac{n_{T}}{2} \mathbb{E}\left[\operatorname { l o g } _ { 2 } \left(1+\rho \operatorname{det}\left(\frac{1}{n_{T}} \mathbf{H}^{H} \mathbf{H}\right)^{1 / n_{T}}\right.\right. \\
\left.\left.+\rho \operatorname{det}\left(\frac{1}{n_{T}} \mathbf{H}_{e}^{H} \mathbf{H}_{e}\right)^{\frac{1}{n_{T}}}\right)\right] .
\end{array}
$$

Since $\mathbf{H}_{e}$ is obtained simply by conjugating and exchanging some elements of the actual matrix $\mathbf{H}$, it can be shown that the

${ }^{1}$ Notation: $\mathbf{A}^{T}, \mathbf{A}^{H}, \mathbf{A}^{*}$ means transpose, hermitian transpose, and complex conjugation, respectively 
eigenvalues of $\left(\mathbf{H}_{e}\right)^{H}\left(\mathbf{H}_{e}\right)$ are the same as the eigenvalues of $\mathbf{H}^{H}(\mathbf{H})$. Therefore, the lower bound is equal to

$R_{s A} \geq \frac{n_{T}}{2} \mathbb{E}\left[\log _{2}\left(1+\rho \exp \ln \left(2 \operatorname{det}\left(\frac{1}{n_{T}} \mathbf{H}^{H} \mathbf{H}\right)^{\frac{1}{n_{T}}}\right)\right)\right]$

Since $\log _{2}\left(1+c e^{x}\right)$ is a convex function in $x$ for $c>0$ and by applying Jensen's inequality it holds that $\mathrm{E}\left[\log _{2}\left(1+c e^{x}\right)\right] \geq$ $\log _{2}(1+c \exp (\mathrm{E}[x]))$, we have

$$
\begin{aligned}
R_{s A} & \geq \frac{n_{T}}{2} \log _{2}\left(1+\rho \exp \mathbb{E}\left[\ln \left(2 \operatorname{det}\left(\frac{1}{n_{T}} \mathbf{H}^{H} \mathbf{H}\right)^{\frac{1}{n_{T}}}\right)\right]\right) \\
& =\frac{n_{T}}{2} \log _{2}\left(1+\rho 2 \exp \frac{1}{n_{T}} \mathbb{E}\left[\ln \left(\operatorname{det}\left(\frac{1}{n_{T}} \mathbf{H}^{H} \mathbf{H}\right)\right)\right]\right) .
\end{aligned}
$$

From [35], [36], we know that

$$
\mathbb{E}\left[\ln \left(\operatorname{det}\left(\frac{1}{n_{T}} \mathbf{H}^{H} \mathbf{H}\right)\right)\right]=\sum_{j=1}^{n_{T}} \mathbb{E}\left[\ln X_{j}\right]-n_{T} \ln n_{T},
$$

where the $X_{j}$ are independent, $\chi^{2}$ distributed independent variables with $2\left(n_{R}-j+1\right)$ degrees of freedom. Using this yields

$$
R_{s A} \geq \frac{n_{T}}{2} \log _{2}\left(1+\frac{\rho}{\frac{n_{T}}{2}} \exp \left(\frac{1}{n_{T}} \sum_{j=1}^{n_{T}} \mathbb{E}\left[\ln X_{j}\right]\right)\right) .
$$

With

$$
\mathbb{E}\left[\ln X_{j}\right]=\psi\left(n_{R}-j+1\right),
$$

where $\psi(\cdot)$ is the digamma function, which may be rewritten for integer arguments as follows

$$
\psi(x)=-\gamma+\sum_{p=1}^{x-1} \frac{1}{p} .
$$

Using this results in the following lower bound for the average rate of the stacked scheme.

$$
\begin{gathered}
R_{s A} \geq \frac{n_{T}}{2} \log _{2}\left(1+\frac{\rho}{\frac{n_{T}}{2}} \exp \left(\frac{1}{n_{T}} \sum_{j=1}^{n_{T}} \sum_{p=1}^{n_{R}-j} \frac{1}{p}-\gamma\right)\right) \\
\quad\left[\text { case } n_{T} \leq n_{R}\right] .
\end{gathered}
$$

Similar steps can be pursued for $n_{T} \geq 4 n_{R}$ resulting in the following lower bound

$$
\begin{gathered}
R_{s A} \geq n_{R} \log _{2}\left(1+\frac{2 \rho}{n_{T}} \exp \left(\frac{1}{2 n_{R}} \sum_{j=1}^{2 n_{R}} \sum_{p=1}^{n_{T} / 2-j} \frac{1}{p}-\gamma\right)\right) \\
{\left[\text { case } n_{T}>4 n_{R}\right]}
\end{gathered}
$$

For the case of $n_{T} \geq 2 n_{R}$ we rewrite (9) as

$$
I_{s A}=\frac{1}{2} \log _{2} \operatorname{det}\left(\mathbf{I}_{2 n_{R}}+\frac{\rho}{n_{T}}\left[\begin{array}{cc}
\mathbf{H H}^{H} & \mathbf{H H}_{e}^{H} \\
\mathbf{H}_{e} \mathbf{H}^{H} & \mathbf{H}_{e} \mathbf{H}_{e}^{H}
\end{array}\right]\right) \text {. }
$$

Since $\mathbb{E}\left[\mathbf{H H}_{e}^{H}\right]=\mathbf{0}$ from proposition 4.1 , we may proceed as in [2] to arrive at a lower bound given as

$$
I_{s A} \geq \frac{1}{2} \sum_{k=1}^{L_{1}} \log _{2}\left(1+\frac{\rho}{n_{T}} X_{k}\right),
$$

where $X_{k}$ are again independent, $\chi^{2}$ distributed independent variables with $2\left(K_{1}-k+1\right)$ degrees of freedom with $K_{1}=$ $\max \left(2 n_{R}, n_{T}\right)$. By following the same line of arguments as in [35], we arrive at

$$
\begin{gathered}
R_{s A} \geq R_{s A}^{l b}=\frac{1}{2} \sum_{j=1}^{L_{1}} \log _{2}\left(1+\frac{\rho}{n_{T}} \exp \left(\sum_{p=1}^{K_{1}-j} \frac{1}{p}-\gamma\right)\right) \\
{\left[\text { case } n_{T} \geq 2 n_{R}\right]}
\end{gathered}
$$

In [23], a similar (however, looser) lower bound was derived for this case in order to analyze the asymptotic performance (with respect to $\rho$ ) of stacked OSTBC.

For the case of $n_{R}<n_{T}<2 n_{R}$ we have

$$
\begin{aligned}
R_{s A} & =\mathbb{E}\left[\frac{1}{2} \log _{2} \operatorname{det}\left(\mathbf{I}+\frac{\rho}{n_{T}}\left(\mathbf{H}^{\prime}\right)^{H} \mathbf{H}^{\prime}\right)\right] \\
& =\mathbb{E}\left[\frac{1}{2} \log _{2} \operatorname{det}\left(\mathbf{I}+\frac{\rho}{n_{T}}\left(\mathbf{H H}^{H}+\mathbf{H}_{e} \mathbf{H}_{e}^{H}\right)\right)\right] \\
& =\mathbb{E}\left[\frac{1}{2} \log _{2} \operatorname{det}\left(\frac{1}{2} \mathbf{I}+\frac{\rho}{n_{T}} \mathbf{H H}^{H}+\frac{1}{2} \mathbf{I}+\frac{\rho}{n_{T}} \mathbf{H}_{e} \mathbf{H}_{e}^{H}\right)\right] .
\end{aligned}
$$

Applying now Minkowski's determinant inequality results in

$$
R_{s A} \geq \frac{1}{2} \mathbb{E}\left[\log _{2} \operatorname{det}\left(\mathbf{I}+\frac{2 \rho}{n_{T}} \mathbf{H H}^{H}\right)\right]
$$

and finally

$$
\begin{aligned}
R_{s A} \geq R_{s A}^{l b}= & \frac{1}{2} \sum_{j=1}^{L} \log _{2}\left(1+\frac{2 \rho}{n_{T}} \exp \left(\sum_{p=1}^{K-j} \frac{1}{p}-\gamma\right)\right) \\
& {\left[\text { case } n_{R}<n_{T}<2 n_{R}\right] . }
\end{aligned}
$$

The lower bound results derived in this subsection are summarized in Table \on the top of the next page.

Note that for high SNR, most of the bounds have a slope equal to $L_{1} / 2$, which equals the slope of the upper bound (11). Only for the case $n_{R}<n_{T}<2 n_{R}$, the slope of the lower bound is equal to $L / 2$. In Fig. 1 on the top of the next page, the average rate, the upper bound (11) and the lower bounds from Table 【 for $n_{T}=$ and $n_{R}=1, \ldots, 4$ are depicted. From the Fig., we observe that the upper bound in (11) and lower bounds track the average rate quite well. Only in the aforementioned case $n_{R}<n_{T}<2 n_{R}$, the slope of the lower bound differs from the exact performance and the upper bound. Note that for $n_{R}=1$, the upper bound coincides with the exact performance.

\section{Characterization of the absolute rate loss}

In this subsection, we characterize the absolute rate loss of the stacked OSTBC to the ergodic capacity using Fischer's inequality. First of all, we discuss the case of $n_{T} \geq 2 n_{R}$. Note that the rate loss with the basic Alamouti scheme $\left(n_{T}=2\right)$ was also analyzed in [8], [10] using different approaches. Starting from (14), applying Fischer's inequality and averaging over 


\begin{tabular}{|c|c|}
\hline Case & Lower bound on $R_{s A}$ \\
\hline$n_{T} \leq n_{R}$ & $\frac{n_{T}}{2} \log _{2}\left(1+\frac{\rho}{\frac{n_{T}}{2}} \exp \left(\frac{1}{n_{T}} \sum_{j=1}^{n_{T}} \sum_{p=1}^{n_{R}-j} \frac{1}{p}-\gamma\right)\right)$ \\
$n_{R}<n_{T}<2 n_{R}$ & $\frac{1}{2} \sum_{j=1}^{L} \log _{2}\left(1+\frac{2 \rho}{n_{T}} \exp \left(\sum_{p=1}^{K-j} \frac{1}{p}-\gamma\right)\right)$ \\
$2 n_{R} \leq n_{T}$ & $\frac{1}{2} \sum_{j=1}^{L_{1}} \log _{2}\left(1+\frac{\rho}{n_{T}} \exp \left(\sum_{p=1}^{K_{1}-j} \frac{1}{p}-\gamma\right)\right)$ \\
$4 n_{R}<n_{T}$ & $n_{R} \log _{2}\left(1+\frac{2 \rho}{n_{T}} \exp \left(\frac{1}{2 n_{R}} \sum_{j=1}^{2 n_{R}} \sum_{p=1}^{n_{T} / 2-j} \frac{1}{p}-\gamma\right)\right)$ \\
\hline
\end{tabular}

TABLE I

LOWER BOUND ON $R_{S A}$ FOR THE DIFFERENT CASES

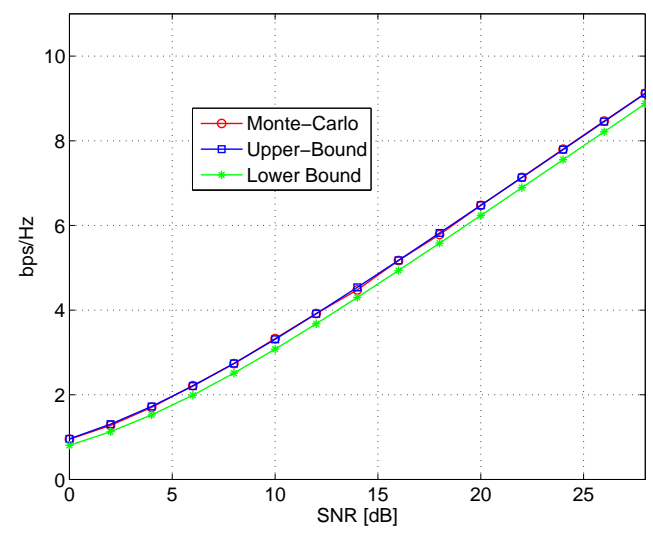

(a) $n_{R}=1$

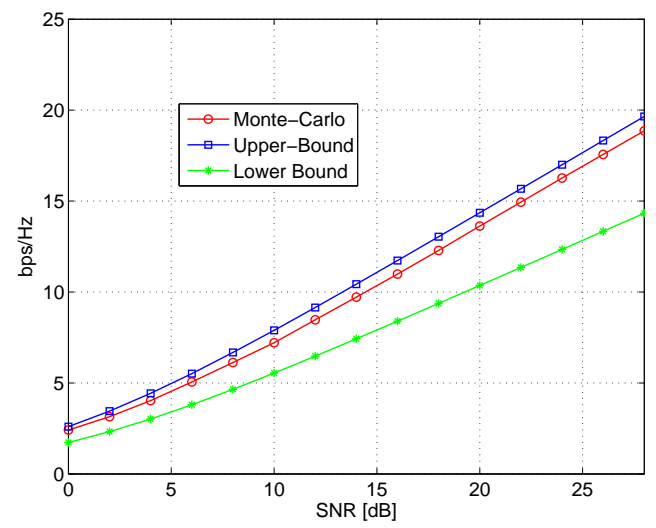

(c) $n_{R}=3$

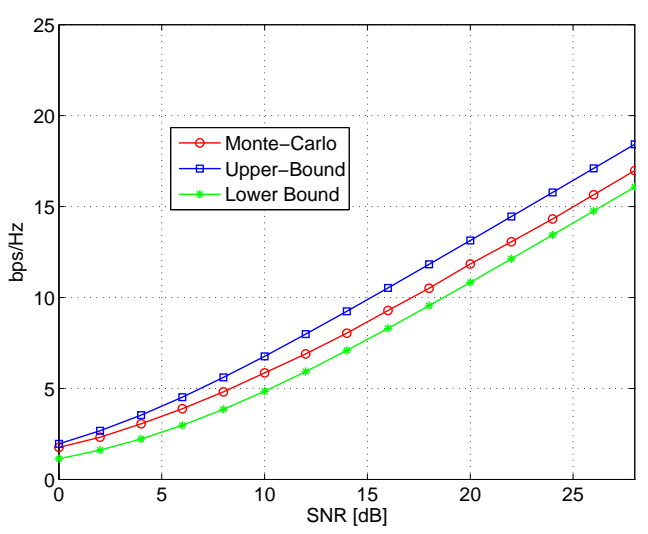

(b) $n_{R}=2$

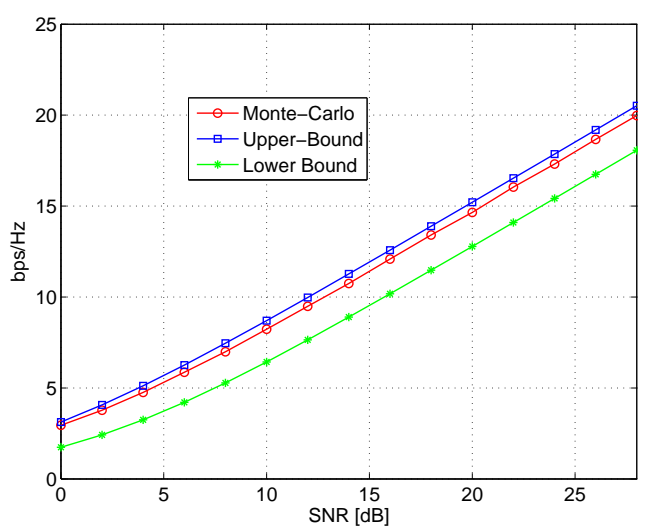

(d) $n_{R}=4$

Fig. 1. Average rates, upper bounds, and lower bounds of the stacked OSTBCs for $n_{T}=4$.

all channel realizations we arrive at

$$
\begin{aligned}
R_{s A} \leq \mathbb{E}[ & \frac{1}{2} \log _{2}\left(\operatorname{det}\left(\mathbf{I}_{n_{R}}+\frac{\rho}{n_{T}} \mathbf{H H}^{H}\right)\right. \\
& \left.\left.\times\left(\mathbf{I}_{n_{R}}+\frac{\rho}{n_{T}} \mathbf{H}_{e} \mathbf{H}_{e}^{H}\right)\right)\right]=C\left(\rho, n_{T}, n_{R}\right)
\end{aligned}
$$

$\left[\right.$ case $\left.n_{T} \geq 2 n_{R}\right]$

i.e. as long as $n_{T} \geq 2 n_{R}$, the average rate of the stacked OSTBC is only upper bounded by the ergodic capacity.
Proceeding similarly for the case $n_{T}<2 n_{R}$ results in

$$
\begin{aligned}
R_{s A} & =\frac{1}{2} \mathbb{E}\left[\log _{2} \operatorname{det}\left(\mathbf{I}_{n_{T}}+\frac{\rho}{n_{T}}\left[\begin{array}{cc}
\tilde{\mathbf{H}}^{H} \tilde{\mathbf{H}} & \tilde{\mathbf{H}}^{H} \tilde{\mathbf{H}}_{e} \\
\tilde{\mathbf{H}}_{e}^{H} \tilde{\mathbf{H}} & \tilde{\mathbf{H}}_{e}^{H} \tilde{\mathbf{H}}_{e}
\end{array}\right]\right)\right] \\
& \leq \mathbb{E}\left[\log _{2}\left(\operatorname{det}\left(\mathbf{I}_{\frac{n_{T}}{2}}+\frac{\rho}{n_{T}} \tilde{\mathbf{H}}^{H} \tilde{\mathbf{H}}\right)\right)\right] \\
& =C\left(\frac{\rho}{2}, \frac{n_{T}}{2}, 2 n_{R}\right)<C\left(\rho, n_{T}, n_{R}\right) \quad\left[\text { case } n_{T}<2 n_{R}\right]
\end{aligned}
$$

where $\tilde{\mathbf{H}}$ is obtained by taking the odd columns of the equivalent channel $\mathbf{H}^{\prime}$ and $\tilde{\mathbf{H}}_{e}$ is obtained by taking the even columns of $\mathbf{H}^{\prime}$. From (17), we observe that for $n_{T}<2 n_{R}$ the average rate of the stacked OSTBC is upper bounded by the ergodic capacity of a system with $\frac{n_{T}}{2}$ transmit and $2 n_{R}$ receive antennas with a power penalty of $3 \mathrm{~dB}$.

We can characterize the gap in (16) and (17) due to the 
application of Fischer's inequality. For $n_{T}<2 n_{R}$, we have then

$$
\begin{aligned}
\Delta & =C\left(\frac{\rho}{2}, \frac{n_{T}}{2}, 2 n_{R}\right)-R_{s A} \\
& =\frac{1}{2} \mathbb{E}\left[\operatorname { l o g } _ { 2 } \left(\frac{\operatorname{det}\left(\mathbf{I}_{n_{T}}+\frac{\rho}{n_{T}} \mathbf{W}_{D}\right)}{\left.\operatorname{det}\left(\mathbf{I}_{n_{T}}+\frac{\rho}{n_{T}}\left[\begin{array}{cc}
\tilde{\mathbf{H}}^{H} \tilde{\mathbf{H}} & \tilde{\mathbf{H}}^{H} \tilde{\mathbf{H}}_{e} \\
\tilde{\mathbf{H}}_{e}^{H} \tilde{\mathbf{H}} & \tilde{\mathbf{H}}_{e}^{H} \tilde{\mathbf{H}}_{e}
\end{array}\right]\right)\right],},\right.\right.
\end{aligned}
$$

where

$$
\mathbf{W}_{D}=\left[\begin{array}{cc}
\tilde{\mathbf{H}}^{H} \tilde{\mathbf{H}} & \mathbf{0} \\
\mathbf{0} & \tilde{\mathbf{H}}_{e}^{H} \tilde{\mathbf{H}}_{e}
\end{array}\right]
$$

Since the events of $\tilde{\mathbf{H}}_{e}^{H} \tilde{\mathbf{H}}$ having not full rank are of measure zero the strict form of Fischer's inequality stated in Lemma 4.1 shows, that the gap in (16) and (17) is non zero in general, i.e. $\Delta>0$, thus it is not possible to reach the upper capacity bounds.

With

$$
\mathbf{W}_{\text {Off }}=\left[\begin{array}{cc}
\tilde{\mathbf{H}}^{H} \tilde{\mathbf{H}} & \tilde{\mathbf{H}}^{H} \tilde{\mathbf{H}}_{e} \\
\tilde{\mathbf{H}}_{e}^{H} \tilde{\mathbf{H}} & \tilde{\mathbf{H}}_{e}^{H} \tilde{\mathbf{H}}_{e}
\end{array}\right]-\mathbf{W}_{D}
$$

we can rewrite

$$
\begin{aligned}
& \operatorname{det}\left(\mathbf{I}_{n_{T}}+\frac{\rho}{n_{T}}\left[\begin{array}{cc}
\tilde{\mathbf{H}}^{H} \tilde{\mathbf{H}} & \tilde{\mathbf{H}}^{H} \tilde{\mathbf{H}}_{e} \\
\tilde{\mathbf{H}}_{e}^{H} \tilde{\mathbf{H}} & \tilde{\mathbf{H}}_{e}^{H} \tilde{\mathbf{H}}_{e}
\end{array}\right]\right) \\
& =\operatorname{det}\left(\mathbf{I}_{n_{T}}+\frac{\rho}{n_{T}}\left(\mathbf{W}_{\text {Off }}+\mathbf{W}_{D}\right)\right) \\
& =\operatorname{det}\left(\mathbf{I}_{n_{T}}+\frac{\rho}{n_{T}} \mathbf{W}_{D}\right) \times \\
& \operatorname{det}\left(\mathbf{I}_{n_{T}}+\left[\mathbf{I}_{n_{T}}+\frac{\rho}{n_{T}} \mathbf{W}_{D}\right]^{-1} \frac{\rho}{n_{T}} \mathbf{W}_{\text {Off }}\right)
\end{aligned}
$$

to arrive at

$$
\Delta=-\frac{1}{2} \mathbb{E}\left[\log _{2} \operatorname{det}(\mathbf{I}_{n_{T}}+\underbrace{\left[\mathbf{I}_{n_{T}}+\frac{\rho}{n_{T}} \mathbf{W}_{D}\right]^{-1} \frac{\rho}{n_{T}} \mathbf{W}_{\text {Off }}}_{\mathbf{A}})\right] .
$$

Using

$$
\operatorname{det}\left(\mathbf{I}_{n_{T}}+\mathbf{A}\right)=\exp \left(\sum_{k=1}^{L_{1}} \ln \left(1+\mu_{k}\right)\right)
$$

yields

$$
\Delta \leq \frac{1}{2 \ln (2)} \mathbb{E}\left[\sum_{k=1}^{L_{1}} \mu_{k}^{2}\right]
$$

where the inequality follows from Taylor series expansion $x-\frac{1}{2} x^{2} \leq \ln (1+x)$ around $x=0$ and the fact that $\operatorname{tr}(\mathbf{A})=0$, since $\mathbf{A}$ has zero block matrices on its diagonal. Its off-diagonal blocks have the form $\mathbf{B}=\left[\mathbf{I}_{n_{T}}+\frac{\rho}{n_{T}} \tilde{\mathbf{H}}^{H} \tilde{\mathbf{H}}\right]^{-1} \frac{\rho}{n_{T}} \tilde{\mathbf{H}}^{H} \tilde{\mathbf{H}}_{\mathbf{e}}$ and $\mathbf{B}_{e}=$ $\left[\mathbf{I}_{n_{T}}+\frac{\rho}{n_{T}} \tilde{\mathbf{H}}_{\mathbf{e}}^{H} \tilde{\mathbf{H}}_{\mathbf{e}}\right]^{-1} \frac{\rho}{n_{T}} \tilde{\mathbf{H}}_{\mathbf{e}}{ }^{H} \tilde{\mathbf{H}}$, respectively. Note that the matrices in brackets have the same eigenvalues. This implies that each eigenvalue of $\mathbf{A}$ appears twice, i.e. $\mu_{k}=\mu_{k+L_{1 / 2}}$, $1 \leq k \leq L_{1} / 2$. Additionally applying the inequality [34,
(5.6.Ex.26)] $\operatorname{tr}\left(\mathbf{A}^{2}\right) \leq\|\mathbf{A}\|^{2}$ we obtain $\sum_{k=1}^{L_{1}} \mu_{k}^{2}=2 \sum_{k=1}^{L_{1} / 2} \mu_{k}^{2} \leq$ $2\|\mathbf{B}\|_{F}^{2}$. Further we have

$$
\|\mathbf{B}\|_{F}^{2}=\operatorname{tr}\left\{\left(\frac{\rho}{n_{T}}\right)^{2} \tilde{\mathbf{H}}_{\mathbf{e}} \tilde{\mathbf{H}}_{\mathbf{e}}{ }^{H} \tilde{\mathbf{H}}\left[\mathbf{I}_{n_{T}}+\frac{\rho}{n_{T}} \tilde{\mathbf{H}}^{H} \tilde{\mathbf{H}}\right]^{-2} \tilde{\mathbf{H}}^{H}\right\}
$$

which can be interpreted as the trace of a product of two positive semi definite matrices $\mathbf{P}, \mathbf{Q}$. Using the fact, that $\tilde{\mathbf{H}}_{\mathbf{e}} \tilde{\mathbf{H}}_{\mathbf{e}}{ }^{H}$ has the same ordered eigenvalues as $\tilde{\mathbf{H}} \tilde{\mathbf{H}}^{H}$ and the inequality $\operatorname{tr}(\mathbf{P Q}) \leq \sum_{j} \mu_{k}(\mathbf{P}) \mu_{k}(\mathbf{Q})$ [37] yields $\sum_{k=1}^{L_{1}} \mu_{k}^{2} \leq$ $L_{1}$ and we arrive at the final bound

$$
\Delta \leq \frac{1}{\ln (2)} \mathbb{E}\left[\sum_{k=1}^{L_{1} / 2} \mu_{k}^{2}\right] \leq \frac{L_{1}}{2 \ln (2)}
$$

In addition to that, we have the loss between $C\left(\frac{\rho}{2}, \frac{n_{T}}{2}, 2 n_{R}\right)$ and $C\left(\rho, n_{T}, n_{R}\right)$. Approximating (7) and (12) for high SNR as

$$
\begin{aligned}
& C_{\text {Jen }}\left(\rho, n_{T}, n_{R}\right)=\log _{2}\left(1+\sum_{i=1}^{L}\left(\begin{array}{c}
L \\
i
\end{array}\right) \frac{K !}{(K-i) !}\left(\frac{\rho}{n_{T}}\right)^{i}\right) \\
\approx & \log _{2}\left(1+\frac{K !}{(K-L) !}\left(\frac{\rho}{n_{T}}\right)^{L}\right) \\
= & \log _{2}\left(\frac{(K-L) !}{K !}+\left(\frac{\rho}{n_{T}}\right)^{L}\right)+\log _{2}\left(\frac{K !}{(K-L) !}\right) \\
\approx & \log _{2}\left(1+\left(\frac{\rho}{n_{T}}\right)^{L}\right) \approx \log _{2}\left(\left(1+\frac{\rho}{n_{T}}\right)^{L}\right) \\
= & L \log _{2}\left(1+\frac{\rho}{n_{T}}\right)
\end{aligned}
$$

and

$$
\begin{aligned}
& C\left(\frac{\rho}{2}, \frac{n_{T}}{2}, 2 n_{R}\right) \\
\stackrel{(a)}{\geq} & \frac{n_{T}}{2} \log _{2}\left(1+\frac{\rho}{n_{T}} \exp \left(\frac{2}{n_{T}} \sum_{j=1}^{\frac{n_{T}}{2}} \sum_{p=1}^{2 n_{R}-j} \frac{1}{p}-\gamma\right)\right) \\
= & \frac{n_{T}}{2} \log _{2}\left(\exp \left(-\frac{2}{n_{T}} \sum_{j=1}^{\frac{n_{T}}{2}} \sum_{p=1}^{2 n_{R}-j} \frac{1}{p}+\gamma\right)+\frac{\rho}{n_{T}}\right) \\
& +\frac{n_{T}}{2 \ln (2)}\left(\frac{2}{n_{T}} \sum_{j=1}^{\frac{n_{T}}{2}} \sum_{p=1}^{2 n_{R}-j} \frac{1}{p}-\gamma\right) \\
\approx & \left(\frac{n_{T}}{2}\right) \log _{2}\left(1+\frac{\rho}{n_{T}}\right),
\end{aligned}
$$

where $(a)$ follows from applying Jensen's inequality to (12). With (18) and (19), the loss between $C\left(\frac{\rho}{2}, \frac{n_{T}}{2}, 2 n_{R}\right)$ and $C\left(\rho, n_{T}, n_{R}\right)$ is quite accurately described by

$$
\begin{aligned}
& C\left(\rho, n_{T}, n_{R}\right)-C\left(\frac{\rho}{2}, \frac{n_{T}}{2}, 2 n_{R}\right) \\
& \approx\left(L-\frac{n_{T}}{2}\right) \log _{2}\left(1+\frac{\rho}{n_{T}}\right), \quad\left[\text { case } n_{T}<2 n_{R}\right] .
\end{aligned}
$$


Finally, the absolute loss for $n_{T}<2 n_{R}$ between the ergodic capacity of a MIMO system and the stacked scheme is given by

$$
\begin{aligned}
& \left(L-\frac{n_{T}}{2}\right) \log _{2}\left(1+\frac{\rho}{n_{T}}\right) \leq C\left(\rho, n_{T}, n_{R}\right)-R_{s A} \\
& \leq \frac{n_{T}}{2 \ln (2)}+\left(L-\frac{n_{T}}{2}\right) \log _{2}\left(1+\frac{\rho}{n_{T}}\right) .
\end{aligned}
$$

The same procedure can be pursued for $n_{T} \geq 2 n_{R}$ resulting in the following general characterization for any $n_{T}, n_{R}$

$$
\begin{gathered}
\max \left(0, L-\frac{n_{T}}{2}\right) \log _{2}\left(1+\frac{\rho}{n_{T}}\right) \leq C\left(\rho, n_{T}, n_{R}\right)-R_{s A} \\
\leq \frac{L_{1}}{2 \ln (2)}+\max \left(0, L-\frac{n_{T}}{2}\right) \log _{2}\left(1+\frac{\rho}{n_{T}}\right),
\end{gathered}
$$

which is equal to

$$
\begin{aligned}
& \left(L-\frac{L_{1}}{2}\right) \log _{2}\left(1+\frac{\rho}{n_{T}}\right) \leq C\left(\rho, n_{T}, n_{R}\right)-R_{s A} \\
& \leq \frac{L_{1}}{2 \ln (2)}+\left(L-\frac{L_{1}}{2}\right) \log _{2}\left(1+\frac{\rho}{n_{T}}\right) .
\end{aligned}
$$

From (20), we observe that as long as $n_{T} \geq 2 n_{R}$, the absolute loss is only a constant, which depends only on the number of receive antennas. In case $n_{T}<2 n_{R}$ the absolute loss increases linearly with $\left(L-\frac{n_{T}}{2}\right)$.

\section{Suboptimal DETECTION AND CONDITION NUMBER}

In the previous sections, we have shown that the stacked OSTBC achieves significant portions of the ergodic capacity. This does not, however, guarantee good performance in terms of error probability, which will be investigated in this section. Note that in the analysis in the previous sections it was implicitly assumed, that an optimal maximum-likelihood detector is used at the receiver, which performs an exhaustive search over all possible transmit symbols at each detection step. Especially for higher number of transmit antennas, this becomes computationally prohibitive. If additionally high rates are requested, then higher order modulation sizes are necessary which increases the computational complexity even more. Thus, suboptimal detection schemes have to be employed reducing the detection complexity and thereby achieving reasonable error rate performance results. Therefore, in this section the impact of the suboptimal LR-aided linear ZFdetector on the performance of the stacked OSTBC is analyzed and compared to SM and QSTBC by resorting the equivalent channel representation. In order to apply the LR algorithm, the system model has to rewritten, which is done in the following subsections for the different transmission schemes. Afterwards, the LR-aided linear ZF-detection is described briefly.

\section{A. Spatial Multiplexing (SM)}

For SM, the transmit matrix $\mathbf{G}_{n_{T}}$ is reduced to $\mathbf{x}$, since $T=1$. In order to apply the suboptimal LR for SM, the system model in (1) has to be rewritten as a real model [28] of the form

$$
\mathbf{y}_{E}=\left[\begin{array}{c}
\Re\{\mathbf{x}\} \\
\Im\{\mathbf{x}\}
\end{array}\right]^{T} \mathbf{H}_{E}^{S M}+\mathbf{n}_{E}
$$

where

$$
\mathbf{y}_{E}=\left[\begin{array}{c}
\Re\{\mathbf{y}\} \\
\Im\{\mathbf{y}\}
\end{array}\right]^{T}, \mathbf{n}_{E}=\left[\begin{array}{c}
\Re\{\mathbf{n}\} \\
\Im\{\mathbf{n}\}
\end{array}\right]^{T}
$$

and

$$
\mathbf{H}_{E}^{S M}=\left[\begin{array}{rr}
\Re\{\mathbf{H}\} & \Im\{\mathbf{H}\} \\
-\Im\{\mathbf{H}\} & \Re\{\mathbf{H}\}
\end{array}\right] .
$$

In the following, we refer to $\mathbf{H}_{E}^{S M}$ as the equivalent channel for the SM scheme.

\section{B. $Q S T B C$}

Without loss of generality, in this subsection we shortly describe the QSTBC for $n_{T}=4$ transmit antennas [38]. To generalization to higher number of transmit antennas is straightforward [16]. The transmit matrix for $n_{T}=4$ transmit antennas is then given [16], [38].

$$
\mathbf{G}_{4}(\mathbf{x})=\left[\begin{array}{rrrr}
x_{1} & x_{2} & x_{3} & x_{4} \\
x_{2}^{*} & -x_{1}^{*} & x_{4}^{*} & -x_{3}^{*} \\
x_{3} & -x_{4} & -x_{1} & x_{2} \\
x_{4}^{*} & x_{3}^{*} & -x_{2}^{*} & -x_{1}^{*}
\end{array}\right] .
$$

After rewriting (1), we arrive at (similar to the proposed scheme, (cf. (2))

$$
\mathbf{y}^{Q}=\mathbf{H}^{Q} \mathbf{x}+\mathbf{n}^{Q},
$$

where $\mathbf{H}^{Q}=\left[\left(\mathbf{H}_{1}^{Q}\right)^{T}, \ldots,\left(\mathbf{H}_{i}^{Q}\right)^{T}, \ldots,\left(\mathbf{H}_{n_{R}}^{Q}\right)^{T}\right]^{T}$ and $\left(\mathbf{H}_{i}^{Q}\right)$ is given as

$$
\mathbf{H}_{i}^{Q}=\left[\begin{array}{rrrr}
h_{1 i} & h_{2 i} & h_{3 i} & h_{4 i} \\
-h_{2 i}^{*} & h_{1 i}^{*} & -h_{4 i}^{*} & h_{3 i}^{*} \\
-h_{3 i} & h_{4 i} & h_{1 i} & -h_{2 i} \\
-h_{4 i}^{*} & -h_{3 i}^{*} & h_{2 i}^{*} & h_{1 i}^{*}
\end{array}\right] .
$$

For general $n_{T}$, we have to rewrite the system model in 21) as a real model similar to SM. For $n_{T}=4$, however, it is not necessary to resort to the real system model. Here, the system model can be decomposed such that the iterative optimal algorithm in [26] for a system with $n_{T}=2$ transmit antennas can be applied. For this we first perform channelmatched filtering as the first stage and noise pre-whitening as the second stage of preprocessing at the receiver resulting in two independent subsystems [39], one of which

$$
\tilde{\mathbf{y}}_{o}=\underbrace{\left[\begin{array}{rr}
\beta & \jmath \beta \\
\epsilon & -\jmath \epsilon
\end{array}\right]}_{\mathbf{H}_{E}^{Q}}\left[\begin{array}{l}
x_{1} \\
x_{3}
\end{array}\right]+\tilde{\mathbf{n}}_{o},
$$

is only a function of the elements of $\mathbf{x}$ with odd index, and the other one is only a function of the elements of $\mathbf{x}$ with even index,

$$
\tilde{\mathbf{y}}_{e}=\underbrace{\left[\begin{array}{rr}
\beta & \jmath \beta \\
\epsilon & -\jmath \epsilon
\end{array}\right]}_{\mathbf{H}_{E}^{Q}}\left[\begin{array}{l}
x_{4} \\
x_{2}
\end{array}\right]+\tilde{\mathbf{n}}_{e},
$$


where $\mathbf{H}_{E}^{Q}$ is the $2 \times 2$ equivalent channel for QSTBC, $\beta=\sqrt{\frac{\lambda+\alpha}{2}}, \epsilon=\sqrt{\frac{\lambda-\alpha}{2}}, \lambda=\sum_{i=1}^{n_{R}} \sum_{j=1}^{n_{T}}\left|h_{i, j}\right|^{2}$, and $\alpha=\sum_{i=1}^{n_{R}} 2 \operatorname{Im}\left(h_{i, 1}^{*} h_{i, 3}+h_{i, 4}^{*} h_{i, 2}\right)$. Both subsystems can now be detected separately, which reduces the complexity of the receiver significantly.

Lemma 5.1: In order to get the best performance with respect to error rates and a decoupled system with scalar input and scalar output as in the case of OSTBC, the columns of $\mathbf{H}_{E}^{Q}$ have to be orthogonal. However, the probability that this occurs for $\mathbf{H}_{E}^{Q}$ is zero.

Proof: For orthogonality, it follows from the scalar product of the columns of $\mathbf{H}_{E}^{Q}$ that $\alpha$ has to be zero. But since the channel entries $\{h j i\}$ are mutually independent and identically distributed (i.i.d.) random complex Gaussian processes, the probability $P_{r}(\alpha=0)$ is equal to the probability $P_{r}\left(\sum_{i=1}^{n_{R}} 2 \operatorname{Im}\left(h_{i, 1}^{*} h_{i, 3}+h_{i, 4}^{*} h_{i, 2}\right)=0\right)$, which in turn is zero. From this it follows that orthogonality and therefore a decoupled system can not be achieved.

A disadvantage of this QSTBC is that in order to achieve the same transmission rate as SM, we have to compensate the rate loss by using a considerably higher constellation. But recall that higher constellations complicates amplification, synchronization, and detection. E.g., a transmission rate of 4 bits $/ \mathrm{sec} / \mathrm{Hz}$ for a system with $n_{T}=4$ transmit antennas is achieved by SM with BPSK, whereas 16QAM is required for the code rate one QSTBC. In [14], [40] it was shown that QSTBC approach the capacity in case of $n_{R}=1$, which is achieved in case of the stacked OSTBC as shown in section IV-A For $n_{R}>1$, the performance of QSTBC in terms of mutual information degrades severely in contrast to the stacked OSTBC, which achieve at least half of the capacity as derived in section IV-B.

\section{Proposed scheme}

Given (2), the equivalent real signal model for the proposed stacked OSTBC is given as

$$
\mathbf{y}^{\prime \prime}=\mathbf{H}_{E}^{O S}\left[\begin{array}{c}
\Re\{\mathbf{x}\} \\
\Im\{\mathbf{x}\}
\end{array}\right]+\mathbf{n}^{\prime \prime}
$$

where

$$
\mathbf{H}_{E}^{O S}=\left[\begin{array}{rr}
\Re\left\{\mathbf{H}^{\prime}\right\} & -\Im\left\{\mathbf{H}^{\prime}\right\} \\
\Im\left\{\mathbf{H}^{\prime}\right\} & \Re\left\{\mathbf{H}^{\prime}\right\}
\end{array}\right] .
$$

\section{LR-aided linear ZF Detection}

By applying the algorithm, the $m \times n$ equivalent channel $\mathbf{H}_{E}$ for each transmission scheme can be decomposed as

$$
\mathbf{H}_{E}=\mathbf{Q R},
$$

where $\mathbf{R}$ is a $n \times n$ matrix with integer entries and $\mathbf{Q}$ is a $m \times n$ matrix, which is better conditioned than $\mathbf{H}_{E}$, i.e. the columns of $\mathbf{Q}$ are less correlated and shorter. A good indication for the correlation of a matrix is the so called condition number, which is defined as the ratio of the largest singular value of the matrix to the smallest. Using (22), the equivalent signal model is then given as

$$
\mathbf{y}=\mathbf{H}_{E} \mathbf{x}_{r}+\mathbf{n}=\mathbf{Q R} \mathbf{x}_{r}+\mathbf{n}=\mathbf{Q z}+\mathbf{n} .
$$

Now, by multiplying $\mathbf{Q}^{-1}$ from left to $\mathbf{y}$ we arrive at

$$
\tilde{\mathbf{y}}=\mathbf{z}+\mathbf{Q}^{-1} \mathbf{n}
$$

where the noise enhancement and coloring is relatively small, since $\mathbf{Q}^{-1}$ is also good conditioned. In order to get a estimation for the transmitted symbols, the following operation has to be applied

$$
\hat{\mathbf{x}}=C\left(\mathbf{R}^{-1} \mathcal{Q}_{\mathbb{Z}^{n}}\left[\frac{1}{C} \tilde{\mathbf{y}}-\mathbf{R} \frac{1}{2} \mathbf{1}_{n}\right]+\frac{1}{2} \mathbf{1}_{n}\right),
$$

where $\mathbf{1}_{n}$ is a $n \times 1$ vector of ones, $C$ is a constant given as $C=\sqrt{\frac{6}{M-1}}$ and $\mathcal{Q}_{\mathbb{Z}^{n}}[\cdot]$ describes the componentwise quantization with respect to the infinite integer space $\mathbb{Z}$. However, this quantization can only be applied, if the transmit modulation signal set $\mathcal{C}$ is transformed to $\mathbb{Z}$, which is achieved with the scaling and shifting of $\tilde{\mathbf{y}}$ within the quantization operation in (23). Note that after this quantization, re-scaling and re-shifting, some points may lie outside the constellation. A suboptimal solution is to assign these points to the nearest point within the constellation. For BPSK, the effect of this assignment has a significant effect on the error rate performance, however, this gain diminishes with higher order modulations.

\section{E. Condition number}

For illustration, the probability density functions (pdfs) of

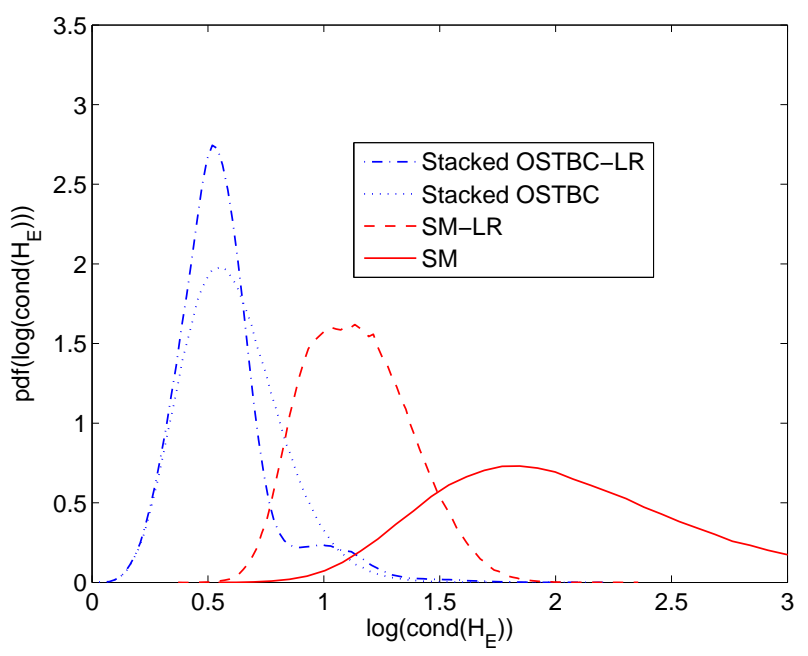

Fig. 2. Pdfs of channel cond. numbers with SM or the stacked OSTBC with and w/o LR for a $4 \times 4$ system.

the natural logarithm of the condition number of the channels for the stacked QSTBC and SM are depicted in Fig. 2. From the Fig., we observe that the SM-channel is bad-conditioned and that LR has a great impact on the channel. For the stacked OSTBC, we observe that the impact of LR is not as significant as for SM.

The pdf of the natural logarithm of the condition number for the QSTBC is depicted in Fig. 3 For comparison, the pdf for the stacked OSTBC is also plotted. In case of QSTBC, for some channels we have no gain with LR, since many samples of the equivalent channel generated with QSTBC have 
inherently low condition numbers such that the LR has no effect. Different from the QSTBC, for the stacked OSTBC there is a gain achieved by applying the LR for almost all samples of the equivalent channel model. Note that for orthogonal channels (e.g., with OSTBC), the pdf is a dirac impulse at position 0 .

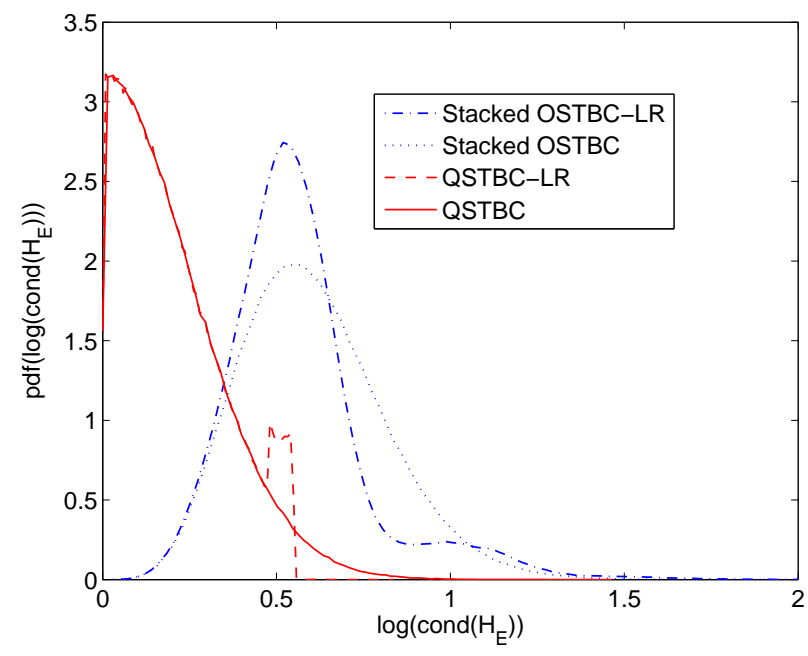

Fig. 3. Pdfs of channel cond. numbers with the stacked OSTBC or QSTBC with and w/o LR.

\section{Simulations}

In Fig. 4, the average rate of the stacked Alamouti scheme and the ergodic capacity of a MIMO system with $n_{R}=2$ and $n_{T}=2,4$ and $n_{T}=8$ is depicted. In case of $n_{T}=2$, we have the standard Alamouti scheme. From the Fig., we observe that the difference between the average rate of the stacked Alamouti scheme and the capacity diminishes significantly by increasing the number of transmit antennas.

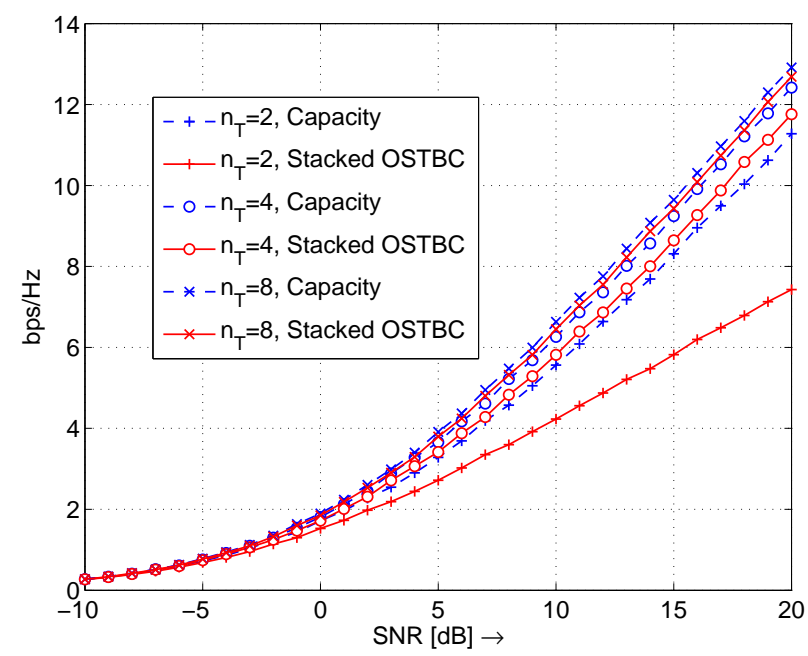

Fig. 4. Ergodic capacity and average rates of the stacked OSTBC with $n_{R}=2$ receive and $n_{T}=2, n_{T}=4$ and $n_{T}=8$ transmit antennas.

In Fig. 5, the average rate of the stacked Alamouti scheme and the ergodic capacity with $n_{T}=4$ and $n_{R}=2,4$ and $n_{T}=$ 8 is depicted. In contrast to the case of increasing number of

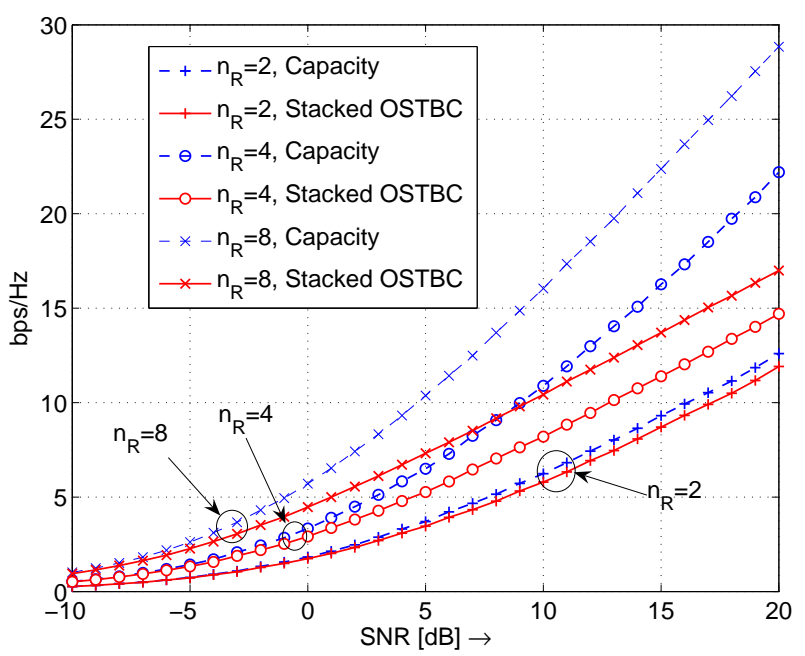

Fig. 5. Ergodic capacity and average rates of the stacked OSTBC with $n_{T}=4$ transmit and $n_{R}=2, n_{R}=4$ and $n_{R}=8$ receive antennas.

transmit antennas, here we observe that the difference between the average rate of the stacked Alamouti scheme and the ergodic capacity increases by increasing the number of receive antennas.

In Fig. 6, the ratio $C / R_{s A}$ is depicted for $n_{T}=8$ transmit and $n_{R}=2$ (bottom) to $n_{R}=9$ (top) receive antennas. For high SNR, we observe that as long as $n_{T} \geq 2 n_{R}$ the ratio decreases as the SNR increases. In case $n_{T}<2 n_{R}$ the ratio increases steadily. As derived in section IV-B, the ratio is upper bounded by $C / R_{s A}<2$ for any $n_{R}, n_{T}$.

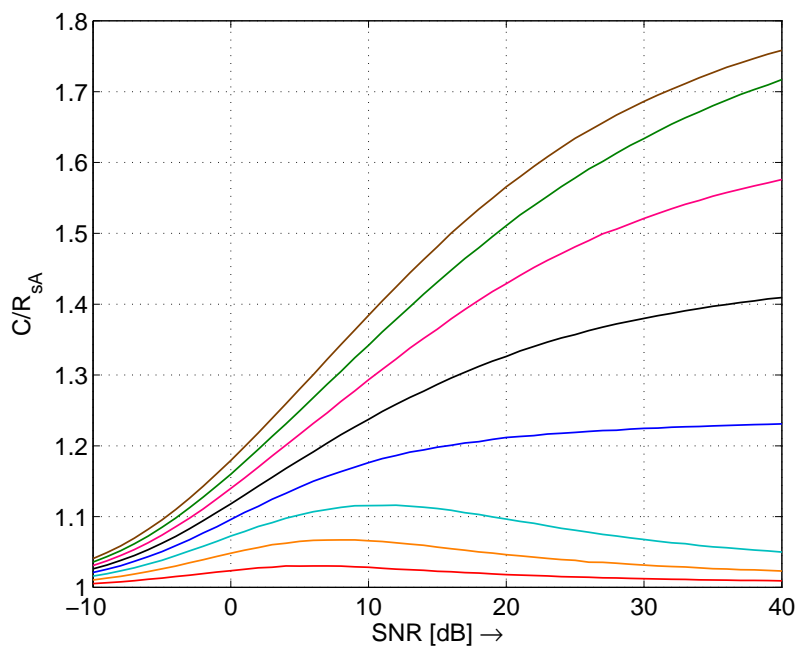

Fig. 6. Ratio $C / R_{s A}$ for $n_{T}=8$ transmit and $n_{R}=2$ (bottom) to $n_{R}=9$ (top) receive antennas.

In Fig. 7, the ratio $C / R_{s A}$ is depicted for $n_{T}=8$ transmit and $n_{R}=4, n_{R}=6$ and $n_{R}=9$ receive antennas. In addition to that, we used our lower and upper bounds derived in the previous section in order to derive lower and upper bounds for the ratio $C / R_{s A}$, i.e.

$$
\frac{C_{l b}}{R_{s A}^{u b}} \leq \frac{C}{R_{s A}} \leq \frac{C_{\mathrm{Jen}}}{R_{s A}^{l b}}
$$


Based on the derivations in section IV-B, we know that the ratio is upper bounded by 2 . Further, since the trivial lower bound is equal to 1 , we only depicted $1 \leq C / R_{s A} \leq 2$. For $n_{R}=9$, we observe that both the lower and upper bound are getting tighter for higher SNR. At low SNR, the upper bound performs better than the lower bound. For $n_{R}=4, n_{R}=6$ and low SNR, we observe that the upper bound is quite loose in comparison to $n_{R}=9$. The lower bound for $n_{R}=4$ is not depicted here, since it is lower than the trivial lower bound of 1.

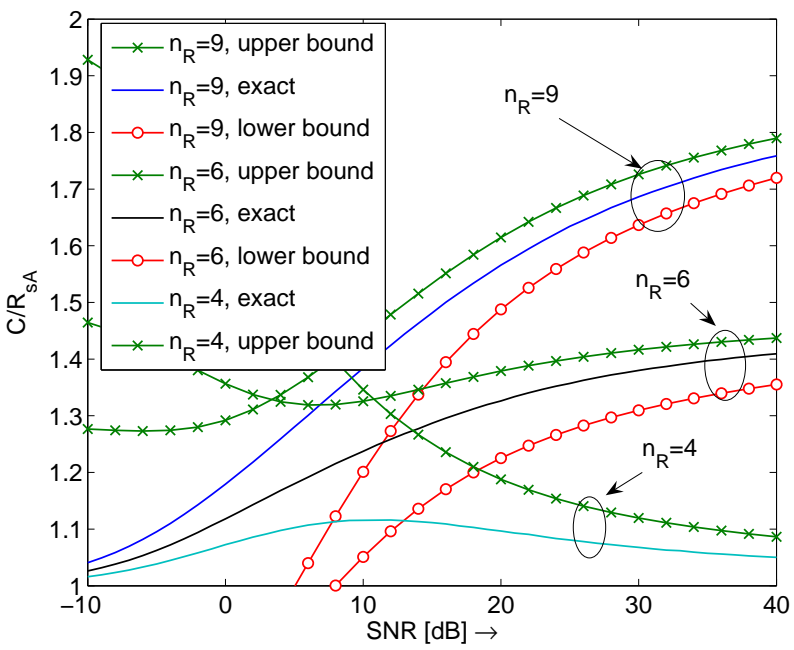

Fig. 7. Ratio $C / R_{s A}$ for $n_{T}=8$ transmit and $n_{R}=4, n_{R}=6$ to $n_{R}=9$ receive antennas.

In Fig. 8, the absolute loss $\Delta$ is depicted for $n_{T}=6$

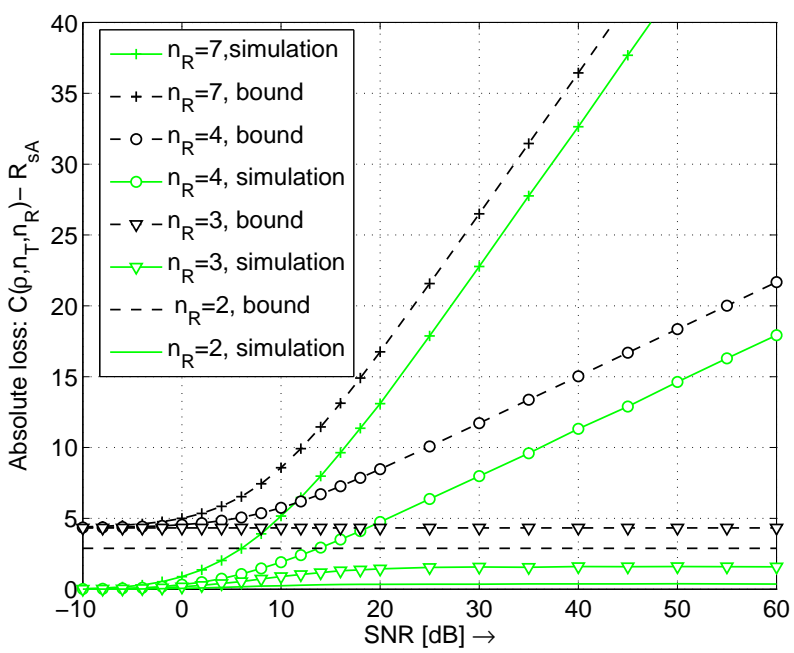

Fig. 8. Absolute loss $\Delta$ for $n_{T}=6$ transmit and different numbers of receive antennas.

transmit antennas and $n_{R}=2-4$ and $n_{R}=7$ receive antennas. From the figure, we observe that as long as $n_{T} \geq$ $2 n_{R}$, the slope of the absolute loss tends to a constant for high SNR. This behavior is tracked quite well by the bound in (20), which is also depicted in the figure.

In Fig. 9, the BER of the stacked OSTBC with QAM and the QSTBC with 16-QAM is depicted for a transmission rate of

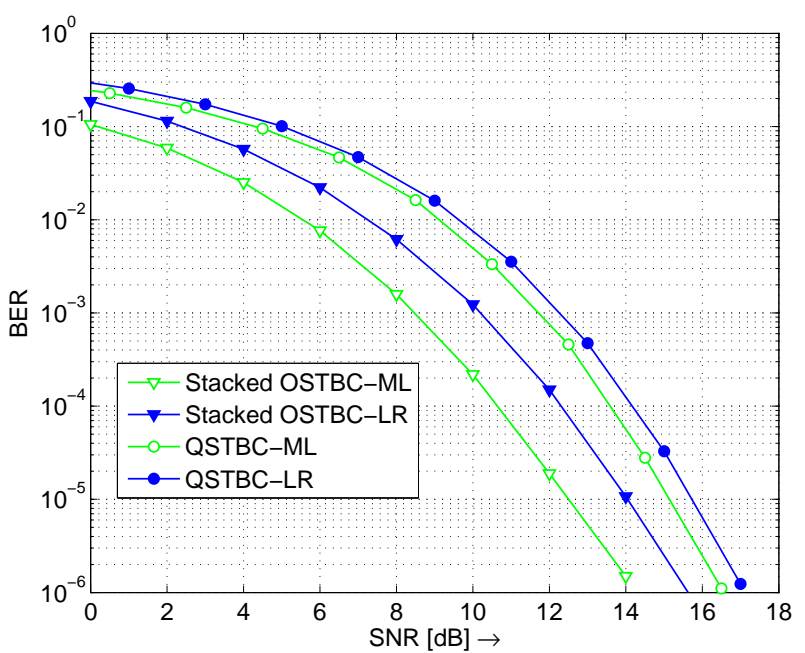

Fig. 9. BER for QSTBC and the stacked OSTBC with ML and LR-ZF, 4 $\mathrm{bit} / \mathrm{sec} / \mathrm{Hz}$

$4 \mathrm{bits} / \mathrm{sec} / \mathrm{Hz}$. Note that in order to make a fair comparison of the three transmission schemes (i.e. QSTBC, SM, and stacked OSTBC), we analyzed a system with $n_{T}=n_{R}=4$ antennas, since for SM with suboptimal detectors it is necessary that $n_{R} \geq n_{T}$. From the figure, we observe, that the performance of the stacked OSTBC with LR-ZF detection is comparable with the optimal ML detection. In fact, the diversity gain of both detectors is equal and there is only a power penalty of about $1.7 \mathrm{~dB}$ of LR-ZF to ML. The gap between ML and LRZF detection is even smaller for QSTBC. Here, the power penalty is about $0.6 \mathrm{~dB}$. Interestingly, the performance of the stacked OSTBC for both ML and LR-ZF detection is better than that of QSTBC in the SNR region shown in the figure. However, for very high SNR and low BER, the diversity gain of $n_{T} n_{R}$ (contrary to diversity of $2 n_{R}$ for the stacked OSTBC) for the QSTBC will show its effect and in can be expected that the performance of QSTBC gets better than that of the stacked OSTBC. For smaller $n_{R}$, this intersection point is expected be at lower SNR values.

The bit error-rate performance of SM for BPSK and a transmission rate of 4 bits $/ \mathrm{sec} / \mathrm{Hz}$ is shown in Fig. 10. For comparison purposes, we also plotted the BER of the stacked QSTBC with QAM. Here, we observe that the BER performance with ML-detection of the stacked OSTBC is better than that of SM for all SNR values. In case of LR-ZF detection, SM performs only better than QSTBC for low SNR of about $2 \mathrm{~dB}$. However, the gap in power efficiency between ML and LR-ZF is higher for the stacked QSTBC in comparison to SM with BSPK. Note that (as aforementioned) the small gap for SM is only due to the BPSK modulation. For higher modulation sizes, this gap is even higher. By increasing the transmission rate to $8 \mathrm{bit} / \mathrm{sec} / \mathrm{Hz}$, i.e. QAM for SM and 16QAM for the stacked OSTBC, we observe in Fig. 11 that the gap between ML and LR-ZF is dramatically increased in case of SM to about $6 \mathrm{~dB}$. On the other hand, the gap between ML and LR-ZF for the stacked OSTBC and 16QAM is reduced in comparison to the gap achieved with QAM (cf. Fig. 10) to about 1.3dB. Although the performance of SM with ML detection is better 


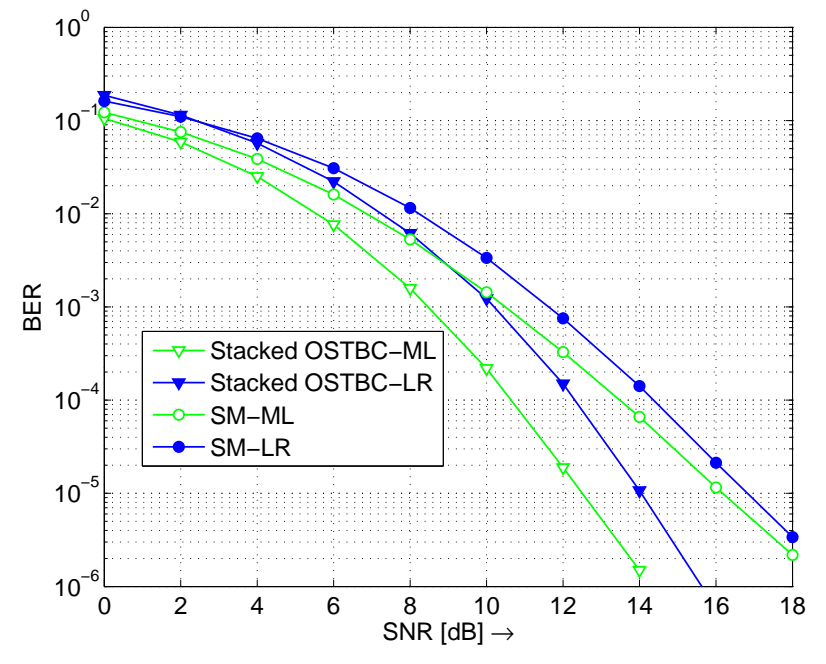

Fig. 10. BER for SM and stacked OSTBC with ML and LR-ZF, 4 bit/sec/Hz.

than that of the stacked OSTBC for low and moderate SNR values, for high SNR values it is the other way around. The

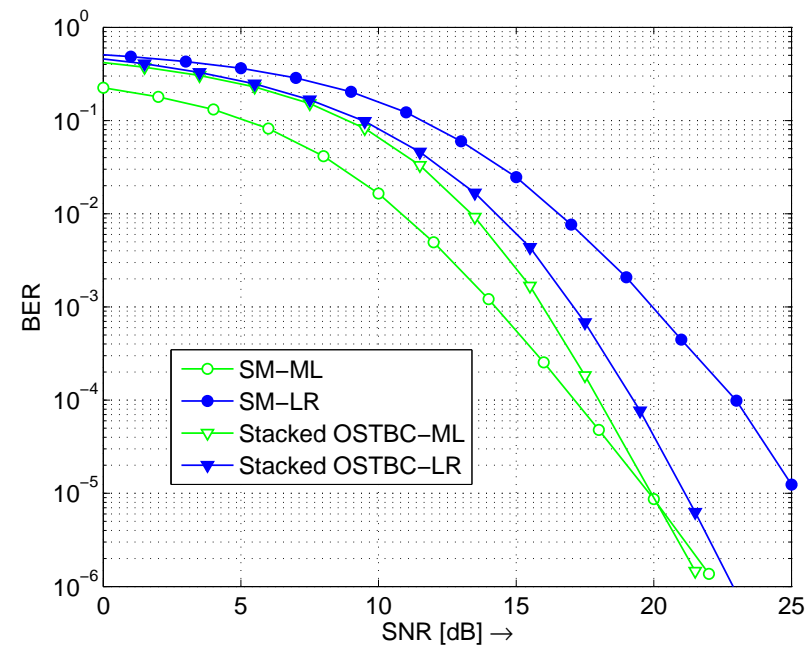

Fig. 11. BER for SM and stacked OSTBC with ML and LR-ZF, 8 bit/sec/Hz.

performance of the stacked OSTBC with LR-ZF detection is better for the whole SNR range in comparison to SM, which is of higher interest for practical applications, since the computational complexity of the ML detector is exponential in the transmission rate. Another disadvantage of SM is that we need at least as many receive as transmit antennas, i.e. $n_{T} \leq n_{R}$, whereas only $\frac{n_{T}}{2}$ receive antennas are necessary for the stacked OSTBC. Multiple receive antennas are only optional for the QSTBC .

\section{CONCLUSION}

In this paper, we analyzed the performance of stacked OSTBC in terms of the average rate. We showed, that the stacked scheme achieves the capacity of a MIMO system in the case of $n_{R}=1$ receive antennas. Further, we showed that the MIMO capacity is at most twice the rate achieved with the proposed scheme at any SNR. We derived lower and upper bounds for the rate achieved with this scheme and compared it with upper and lower bounds for the capacity.

In addition to the capacity analysis, we also analyzed the error rate performance of the proposed scheme. To this end, we combined the stacked OSTBC with a zero-forcing (ZF) detector applying lattice-reduction (LR) aided detection, since this suboptimal detector achieves the same diversity as the optimal ML detector with only some penalty in power efficiency. We analyzed the effect of LR on the equivalent channel generated by the stacked OSTBC, for spatial multiplexing (SM) and QSTBC. We observed the highest gain for SM and a higher gain for the stacked OSTBC in comparison to the QSTBC.

Finally, we illustrated the theoretical results by numerical simulations. From simulation results we observed that the stacked scheme approaches the ergodic capacity of a MIMO system by increasing the number of transmit antennas for a fixed number of receive antennas. Furthermore, we observed that as long as the number of transmit antennas is twice the number of receive antennas the ratio of the capacity to the rate of the proposed scheme improves by increasing the SNR. Regarding the simulation of the error rate performance, we observed that in the considered SNR region the stacked OSTBC performs better in terms of BER for ML as well as for LR-aided ZF-detection than SM and QSTBC in the setup given. Further, we observed that the gap between maximumlikelihood and LR-ZF detection is dramatically reduced in comparison to SM schemes, especially for higher transmission rates.

\section{ACKNOWLEDGEMENTS}

The authors thank the reviewers for their detailed and insightful comments, which significantly enhanced the quality and readability of the paper.

\section{REFERENCES}

[1] E. Telatar, "Capacity of multi-antenna Gaussian channels," European Trans. on Telecomm. ETT, vol. 10, no. 6, pp. 585-596, November 1999.

[2] G. Foschini and M. Gans, "On limits of wireless communications in a fading environment when using multiple antennas," Wireless Personal Communications, vol. 6, no. 3, pp. 311-335, March 1998.

[3] A. Hottinen, O. Tirkkonen, and R. Wichman, Multi-antenna Transeiver Techniques for $3 G$ and Beyond. Wiley, 2003.

[4] V. Tarokh, N. Seshadri, and A. Calderbank, "Space-time codes for high data rate wireless communication:performance criterion and code construction," IEEE Trans. on Information Theory, vol. 44, no. 2, pp. 744-765, March" 1998.

[5] S. Alamouti, "A simple transmitter diversity scheme for wireless communications," IEEE Journal on Selected Areas in Communications, vol. SAC-16, pp. 1451-1458, October 1998.

[6] V. Tarokh, H. Jafarkhani, and A. Calderbank, "Space-time block codes from orthogonal designs," IEEE Trans. on Information Theory, vol. 45, no. 5, pp. 1456-1467, July 1999.

[7] G. Foschini, "Layered space-time architecture for wireless communication in a fading environment when using multi-element antennas," Bell Labs Tech. J., vol. 1, no. 2, pp. 41-59, Autumn 1996.

[8] B. Hassibi and B. Hochwald, "High-rate codes that are linear in space and time," IEEE Trans. on Information Theory, vol. 48, no. 7, pp. 1804 -1824 , July 2002.

[9] R. Nabar, H. Bölcskei, and A. Paulraj, "Diversity and outage performance in Ricean MIMO channels," IEEE Trans. Wireless Communications, vol. 4, no. 5, pp. 2519 - 2532, September 2005.

[10] S. Sandhu and A. Paulraj, "Space-time block codes: A capacity perspective," IEEE Comm. Letters, vol. 4, no. 12, pp. 384 -386, December 2000. 
[11] G. Bauch and J. Hagenauer, "Smart versus dumb antennas-capacities and FEC performance," IEEE Comm. Letters, vol. 6, no. 2, pp. 55-57, February 2002.

[12] E. Jorswieck, A. Sezgin, and H. Boche, "Outage probability of OSTBC: Optimal transmit strategy and suboptimality of odd number of transmit antennas," will be presented at ICASSP 2006, Toulouse, France, May 15-19 2006

[13] H. Jafarkhani, "A quasi-orthogonal space-time block code," IEEE Trans. on Comm., vol. 49, no. 1, pp. 1-4, January 2001.

[14] C. Papadias and G. Foschini, "Capacity-approaching space-time codes for systems employing four transmit antennas," IEEE Trans. on Info. Theory, vol. 49, no. 3, pp. 726 -733, March 2003.

[15] O. Tirkkonen, A. Boariu, and A. Hottinen, "Minimal non-orthogonality rate 1 space-time block code for 3+ Tx antennas," IEEE ISSSTA 2000, pp. 429-432, September 2000.

[16] A. Sezgin and T. Oechtering, "On the outage probability of quasiorthogonal space-time codes," Proc. of IEEE Info. Theory Workshop 2004, San Antonio, TX, USA, October 2004.

[17] - "Complete characterization of the equivalent MIMO Channel for quasi-orthogonal space-time codes," submitted to IEEE Transactions on Information Theory. Also available at http://www.user.tu-berlin.de/sezgijdi/ 2004.

[18] V. Tarokh, A. Naguib, N. Seshadri, and A. Calderbank, "Combined array processing and space-time coding," IEEE Trans. on Info. Theory, vol. 45, no. 4, pp. 1121-1128, May 1999.

[19] A. Naguib, N. Seshadri, and A. Calderbank, "Applications of space-time block codes and interference suppression for high data rate wireless systems," Proc. Asilomar Conf. on Signals, Systems and Computers, pp. 1803-1810, 1998.

[20] N. Prasad and M. Varanasi, "Optimum efficiently decodable layered space-time block codes," Proc. Asilomar Conf. on Signals, Systems, and Computers, Montery, CA, November 2001.

[21] _ - "Outage analysis and optimization of a stacked orthogonal spacetime architecture and near-outage codes," Proc. Commun. Th. Symp. IEEE GLOBECOM, San Francisco, CA, USA, December 2003.

[22] A. Sezgin and E. Jorswieck, "Capacity achieving high rate space-time block codes," IEEE Communications Letters, vol. 9, no. 5, pp. 435-437, May 2005.

[23] Y. Li and Z. Ye, "On the capacity of stacked orthogonal space-time architecture over mimo channels," in Proc. of Intern. Conf. on Comm., Circuits and Systems, vol. 1, pp. 279-282, May 27-30 2005.

[24] T. Instruments, "Double-STTD scheme for HSDPA systems with four transmit antennas: Link level simulation results," TSG-R WG1 document, TSGR1\# 20(01)0458, May 21-24, Busan, Korea 2001.

[25] P. Wolniansky, G. Foschini, D. Golden, and R. Valenzuela, "V-BLAST: An architecture for realizing very high data rates over the rich-scattering wireless channel," PROC. ISSSE, 1998.

[26] H. Yao and G. Wornell, "Lattice-reduction-aided detectors for MIMO communication systems," Proc. of IEEE Globecom 2002, Taipei, Taiwan, November 2002.

[27] Q. Meng, Z. Pan, X. You, and Y. Kim, "On performance of lattice reduction aided detection in the presence of receive correlation," Proc. of IEEE 6th Circuits and Systems Symposium on Emerging Technologies: Frontiers of Mobile and Wireless Communication, 2004, vol. 1, pp. 8992, 2004.

[28] C. Windpassinger and R. Fischer, "Low-complexity near-maximumlikelihood detection and precoding for mimo systems using lattice reduction," Proc. of IEEE ITW 2003, Paris, France, April 2003.

[29] A. Lenstra, H. Lenstra, and L. Lovász, "Factoring palynomials with rational coefficients," Math. Ann., vol. 261, pp. 515-534, 1982.

[30] D. Wübben, R. Böhnke, V. Kühn, and K. Kammeyer, "Near-maximumlikelihood detection of MIMO systems using MMSE-based lattice reduction," Proc. of IEEE ICC 2004, Paris, France, June 2004.

[31] I. Gradshteyn and I. Ryzhik, Table of Integrals, Series, and Products, 4th ed. Academic Press, Inc., 1983.

[32] A. Grant, "Rayleigh fading multi-antenna channels," EURASIP J. Appl. Signal Processing (Special Issue on Space-Time Coding (Part I)), vol. 2002, no. 3, pp. 316-329, March 2002.

[33] O. Henkel and G. Wunder, "Space frequency codes from sphere packings," in Proceedings International ITG/IEEE Workshop on Smart Antennas (WSA 2005), 2005.

[34] R. Horn and C. Johnson, Matrix Analysis. Cambridge University Press, 1985.

[35] Ö. Oyman, R. Nabar, H. Bölcskei, and A. Paulraj, "Characterizing the statistical properties of mutual information in MIMO channels," IEEE TRANSACTIONS ON SIGNAL PROCESSING, vol. 51, no. 11, pp. 2784 -2795 , November 2003.
[36] N. Goodman, "The distribution of the determinant of a complex wishart distributed matrix," Ann. Math. Stat., vol. 34, no. 1, pp. 178 -180, Mar. 1963.

[37] A. Marshall and I. Olkin, Inequalities: Theory of Majorization and Its Applications, ser. Mathematic in Science and Engineering. Academic Press, 1979, vol. 143.

[38] N. Sharma and C. Papadias, "Improved quasi-orthogonal codes," IEEE Wireless Comm. and Network Conf., Orlando, FL, USA, pp. $169-171$, 17-21 March 2002.

[39] - "Improved quasi-orthogonal codes through constellation rotation," IEEE Trans. on Comm., vol. 51, no. 3, pp. 332 -335, March 2002.

[40] A. Sezgin and O. Henkel, "Capacity, BER and coding gain analysis for rate one QSTBC: A general approach," ASILOMAR CSSC 2005, Pacific Grove, CA USA, October 30 - November 22005. 